\title{
Comparative analysis of effects of dietary arachidonic acid and EPA on growth, tissue fatty acid composition, antioxidant response and lipid metabolism in juvenile grass carp, Ctenopharyngodon idellus
}

\author{
Jing-jing Tian ${ }^{1,2}$, Cai-xia Lei ${ }^{1}$, Hong $\mathrm{Ji}^{1 *}$, Gen Kaneko ${ }^{3}$, Ji-shu Zhou ${ }^{1}$, Hai-bo $\mathrm{Yu}^{1}$, Yang Li $^{1}$, Er-meng Yu ${ }^{2}$ \\ and Jun Xie ${ }^{2}$ \\ ${ }^{1}$ College of Animal Science and Technology, Northwest AEF University, Yangling 712100, People's Republic of China \\ ${ }^{2}$ Key Laboratory of Tropical \& Subtropical Fishery Resource Application \& Cultivation, Ministry of Agriculture, Pearl River \\ Fisheries Research Institute, Chinese Academy of Fishery Sciences, Guangzhou 510380, People's Republic of China \\ ${ }^{3}$ School of Arts \& Sciences, University of Houston-Victoria, Victoria, TX 77901, USA \\ (Submitted 24 April 2017 - Final revision received 26 July 2017 - Accepted 27 July 2017)
}

\section{Abstract}

Four isonitrogenous and isoenergetic purified diets containing free arachidonic acid (ARA) or EPA (control group), 0.30\% ARA, 0.30\% EPA and $0 \cdot 30 \%$ ARA + EPA (equivalent) were designed to feed juvenile grass carp (10.21 (sD 0.10) g) for 10 weeks. Only the EPA group presented better growth performance compared with the control group $(P<0 \cdot 05)$. Dietary ARA and EPA were incorporated into polar lipids more than non-polar lipids in hepatopancreas but not intraperitoneal fat (IPF) tissue. Fish fed ARA and EPA showed an increase of serum superoxide dismutase and catalase activities, and decrease of glutathione peroxidase activity and malondialdehyde contents $(P<0 \cdot 05)$. The hepatopancreatic TAG levels decreased both in ARA and EPA groups $(P<0.05)$, accompanied by the decrease of lipoprotein lipase (LPL) activity in the ARA group $(P<0.05)$. Fatty acid synthase $(F A S)$, diacylglycerol $O$-acyltransferase and $a p o E$ gene expression in the hepatopancreas decreased in fish fed ARA and EPA, but only the ARA group exhibited increased mRNA level of adipose TAG lipase ( $A T G L$ ) $(P<0 \cdot 05)$. Decreased IPF index and adipocyte sizes were found in the ARA group $(P<0 \cdot 05)$. Meanwhile, the ARA group showed decreased expression levels of adipogenic genes CCAAT enhancer-binding protein $\alpha, L P L$ and $F A S$, and increased levels of the lipid catabolic genes $P P A R \alpha, A T G L$, hormone-sensitive lipase and carnitine palmitoyltransferase $1(C P T-1)$ in IPF, whereas the EPA group only increased PPAR $\alpha$ and CPT-1 mRNA expression and showed less levels than the ARA group. Overall, dietary EPA is beneficial to the growth performance, whereas ARA is more potent in inducing lipolysis and inhibiting adipogenesis, especially in IPF. Meanwhile, dietary ARA and EPA showed the similar preference in esterification and the improvement in antioxidant response.

Key words: Long-chain PUFA: Principle component analysis: Antioxidant status: Lipid metabolism: Ctenopharyngodon idellus

Long-chain PUFA (LC-PUFA), such as EPA $(20: 5 n-3)$, docosapentaenoic acid (DPA, 22:5n-3), DHA (22:6n-3) and arachidonic acid (ARA, $20: 4 n-6$ ), play unique roles in controlling and regulating growth performance, cell membrane fluidity, lipid metabolism and immune function in fish ${ }^{(1-4)}$. Among these fatty acids, ARA and EPA are the two main $\mathrm{C}_{20}$ LC-PUFA, which are the precursors for one of the most important groups of bioactive lipid mediators, the eicosanoids ${ }^{(5-7)}$. There exists three enzymatic routes for the synthesis of eicosanoids: the cyclo-oxygenase (COX) pathway, the lipoxygenase (LOX) pathway and the cytochrome 450 pathway ${ }^{(8,9)}$. ARA shows similar structure with EPA but lacks one double bond located at the third carbon from the $n$ end, and it is believed that these two fatty acids compete for binding to the eicosanoid synthesis enzymes such as COX or LOX $^{(6,10)}$. In mammals, ARA is the chief precursor of eicosanoids, which generate two-series prostanoids (PG) and 4-series leukotrienes (LT), whereas EPA converted to 3-series PG and 5-series LT; these metabolites from EPA are generally less biologically active than the corresponding metabolites from $\operatorname{ARA}^{(6,11)}$. However, some recent studies have shown a similar activity of ARA-derived $\mathrm{PGE}_{2}$ and EPA-derived $\mathrm{PGE}_{3}$ such as in endothelial cells $^{(12,13)}$. Moreover, the sn-2 position of fish phosphoinositides (PI) has a distinct preference for $\mathrm{C}_{20}$ PUFA, and the existence of a competition between ARA and EPA during phospholipid esterification has also been suggested, especially in $\mathrm{PI}^{(11,14)}$. However, ARA and EPA are also very susceptible to be attacked by $\mathrm{O}_{2}$

Abbreviations: ARA, arachidonic acid; ATGL, adipose TAG lipase; CAT, catalase; COX, cyclo-oxygenase; FAS, fatty acid synthase; GSH-Px, glutathione peroxidase; IPF, intraperitoneal fat; LC-PUFA, long-chain PUFA; LPL, lipoprotein lipase; MDA, malondialdehyde; NPL, non-polar lipids; PL, polar lipids; SOD, superoxide dismutase.

* Corresponding author: Professor H. Ji, fax +86 029 87092585, email jihong@nwsuaf.edu.cn 
radicals and other organic radicals, resulting in serious consequences for cell membrane structure and fluidity ${ }^{(6)}$. Several enzyme systems exist for the possible protection of membrane LC-PUFA in fish, such as superoxide dismutase (SOD), catalase (CAT) and glutathione peroxidase (GSH-Px) ${ }^{(6)}$

Studies have shown that ARA has unique roles in regulating stress resistance ${ }^{(15-18)}$, immune response ${ }^{(19-21)}$, reproductive performance $^{(22-24)}$, pigmentation ${ }^{(25-27)}$, morphogenesis ${ }^{(28,29)}$ and fatty acid metabolism ${ }^{(30,31)}$ of fish. Recently, we have shown that moderate dietary ARA $(0 \cdot 30 \%)$ decreased lipid accumulation and lipogenic gene expression levels in grass carp (Ctenopharyngodon idellus) ${ }^{(4)}$. It has already been further demonstrated that the COX pathway is responsible for the improvement of lipid catabolic genes rather than lipogenic genes by using COX inhibitor acetylsalicylic $\operatorname{acid}^{(32,33)}$. In addition, it has also been demonstrated that $n-3$ LC-PUFA, including EPA, could decrease the lipid deposition and increase the capacity of lipolysis in this fish species $^{(3,34,35)}$. These studies indicated that both $n$ - 6 and $n$-3 LC-PUFA played peculiar roles in regulating lipid metabolism of grass carp, whereas the efficacy of these two fatty acids, such as ARA and EPA, remains largely unknown.

Grass carp, a typical herbivorous freshwater fish species that is widely cultured in China, easily accumulates excessive fat in the abdominal cavity and suffers from fatty liver in aquaculture $^{(32,36)}$. It is worth noting that grass carp could synthesise LC-PUFA from linoleic acid (LA) and $\alpha$-linolenic acid (LNA) via its desaturase and elongase multienzyme complexes ${ }^{(37,38)}$. ARA is an end product of the $n-6$ series fatty acids, whereas EPA is easily converted to DHA, which is the end product of the $n$ - 3 series fatty acids ${ }^{(6,39)}$. Thus, ARA and EPA should have different patterns for deposition in tissues of this fish species, which might further differently affect physiological functions, such as lipid metabolism. Therefore, the aim of this study was to compare the efficacy of dietary ARA and EPA on the lipid accumulation in grass carp. Four purified diets containing different fatty acids (ARA or EPA) were designed to feed grass carp for 10 weeks, and the growth, tissue fatty acid composition, antioxidant response and lipid metabolism were evaluated, with the purpose of providing reference for the use of these two fatty acids in aquaculture industry.

\section{Material and methods}

\section{Experimental diets}

Four isonitrogenous and isoenergetic semipurified diets containing $36.0 \%$ crude protein and $6.0 \%$ crude lipid were formulated, based on the method of Lavell with some modifications ${ }^{(40)}$. Soyabean oil (Kerry Oils \& Grains Co.) and linseed oil (Hoval Seasons Bio-Sci Co.) were added to satisfy the $1 \%$ LA and $1 \%$ LNA requirements, respectively ${ }^{(37)}$ (Table 1). The control diet was ARA-free, whereas the other three diets were added with ARA-enriched oil (ARA content, approximately $40.8 \%$ of total fatty acid; in the form of ARA-methylester; Hubei Fuxing Biotechnology Co., Ltd) or EPA-enriched oil (EPA content, approximately $62 \%$ of total fatty acid; Xunda Marine Bio-Pro Co.) at the expense of lard oil (purchased from
Table 1. Formulation and chemical composition of the experimental diets (g/kg DM)

\begin{tabular}{|c|c|c|c|c|}
\hline \multirow[b]{2}{*}{ Components } & \multicolumn{4}{|c|}{ Groups } \\
\hline & Control & ARA & EPA & $\mathrm{ARA}+\mathrm{EPA}$ \\
\hline Casein & 320 & 320 & 320 & 320 \\
\hline Gelatin & 80 & 80 & 80 & 80 \\
\hline Dextrin & 280 & 280 & 280 & 280 \\
\hline Cellulose & 189 & 189 & 188 & 188 \\
\hline Soyabean oil & 11 & 11 & 11 & 11 \\
\hline Linseed oil & 19 & 19 & 19 & 19 \\
\hline ARA-enriched oil & 0 & $7 \cdot 5$ & 0 & 3.75 \\
\hline EPA-enriched oil & & & 5 & 2.5 \\
\hline Lard oil & 30 & $22 \cdot 5$ & 25 & 23.75 \\
\hline Carboxymethylcellulose & 20 & 20 & 20 & 20 \\
\hline Mineral mix ${ }^{*}$ & 40 & 40 & 40 & 40 \\
\hline Vitamin mix† & 10 & 10 & 10 & 10 \\
\hline $\mathrm{BHT}$ & 1 & 1 & 1 & 1 \\
\hline Total & 1000 & 1000 & 1000 & 1000 \\
\hline \multicolumn{5}{|l|}{ Chemical composition } \\
\hline Moisture (\%) & $10 \cdot 33$ & $10 \cdot 19$ & $10 \cdot 16$ & $10 \cdot 22$ \\
\hline Crude protein $(\%, \mathrm{~N} \% \times 6.25)$ & $36 \cdot 12$ & 35.98 & 35.63 & 35.45 \\
\hline Crude fat $(\%)$ & $5 \cdot 92$ & $5 \cdot 90$ & 5.99 & 5.96 \\
\hline Ash (\%) & $5 \cdot 45$ & 5.55 & 5.59 & 5.52 \\
\hline
\end{tabular}

ARA, arachidonic acid; BHT, butylated hydroxytoluene.

* The mineral mix contained the following $\left(\mathrm{g} / 100 \mathrm{~g}\right.$ of the total mineral): $\mathrm{KAl}\left(\mathrm{SO}_{4}\right)$, 0.159; $\mathrm{CaCO}_{3}, 18 \cdot 101 ; \mathrm{Ca}\left(\mathrm{H}_{2} \mathrm{PO}_{4}\right)_{2}, 44.601 ; \mathrm{CoCl}, 0.070 ; \mathrm{MgSO}_{4}, 5.216 ; \mathrm{MnSO}_{4}$. $\mathrm{H}_{2} \mathrm{O}, 0.070 ; \mathrm{KCl}, 16.553 ; \mathrm{KI}, 0.014 ; \mathrm{ZnCO}_{3}, 0.192 ; \mathrm{NaH}_{2} \mathrm{PO}_{4}, 13.605 ; \mathrm{Na}_{2} \mathrm{SeO}_{3}$, $0.006 ; \mathrm{CuSO}_{4} .5 \mathrm{H}_{2} \mathrm{O}, 0.075$; ferric citrate, 1.338 .

† The vitamin mix contained the following $(\mathrm{mg} / 1000 \mathrm{~g}$ of diet): vitamin C, 200; thiamine, 10; riboflavin, 20; vitamin $A, 7.5$; vitamin $E, 500$; vitamin $D_{3}, 0.5$; menadione, 10; pyridoxine $\mathrm{HCl}, 10$; cyanocobalamin, 0.02; biotin, 1.0; calcium pantothenate, 40; folic acid, 5; niacin, 20; inositol, 400; choline chloride, 2000; and cellulose was used as a carrier.

Kangle Market) to meet the $0 \cdot 30 \%$ ARA content, $0 \cdot 30 \%$ EPA content and $0.30 \%$ ARA + EPA (equivalent), respectively. A measure of $0 \cdot 1 \%$ butylated hydroxytoluene (Sigma-Aldrich) was added as the antioxidant (Table 1). The fatty acid compositions of the experimental diets were determined by GC and are given in Table 2 .

Ingredients were mixed by hand and approximately $70 \%$ water was added to make a dough, and the dough was then pressed to obtain noodle-like pellets with a diameter of $2 \mathrm{~mm}$. The pellets were dried under forced air at room temperature for $24 \mathrm{~h}$, which were then kept at $-20^{\circ} \mathrm{C}$ until use.

\section{Experimental procedure}

Juvenile grass carps were obtained from the Ankang Fisheries Experimental and Demonstration Station of Northwest A\&F University (Ankang, China). Fish were reared in the aquaria and fed a commercial diet (protein 36.54\%, fat $4.99 \%$ dry weight and soyabean oil as a lipid source) for two weeks to acclimatise to the experimental condition.

Before the feeding experiment, fish were fasted for $24 \mathrm{~h}$. A total of 216 fish (10.21 (sD 0.10) g body weight) were randomly distributed into twelve aquaria $(0.73 \times 0.46 \times 0.60 \mathrm{~m} ; 18 \mathrm{fish} /$ aquarium). Each diet was randomly assigned into three aquaria. Fish were hand-fed to apparent satiation twice daily (at 09.00 and 16.00 hours) for 10 weeks. Satiation feeding was achieved by allowing fish to eat until feeding activity stopped, with no feed remaining in the aquaria. The feed intake was recorded. 
Table 2. Fatty acid composition of the experimental diets (percentage of total fatty acids)

\begin{tabular}{|c|c|c|c|c|}
\hline \multirow[b]{2}{*}{ Fatty acids } & \multicolumn{4}{|c|}{ Groups } \\
\hline & Control & ARA & EPA & $A R A+E P A$ \\
\hline $14: 0$ & 1.62 & 1.36 & 1.45 & 1.45 \\
\hline $16: 0$ & $19 \cdot 72$ & $17 \cdot 76$ & 17.89 & $17 \cdot 80$ \\
\hline $18: 0$ & 13.64 & 12.07 & $12 \cdot 30$ & $12 \cdot 21$ \\
\hline SFA & 34.97 & $31 \cdot 18$ & 31.63 & 31.45 \\
\hline $16: 1 n-7$ & 1.04 & 0.72 & 0.93 & 0.86 \\
\hline $18: 1 n-9$ & $27 \cdot 16$ & 24.71 & 24.90 & $25 \cdot 14$ \\
\hline MUFA & $28 \cdot 20$ & 25.43 & $25 \cdot 83$ & $26 \cdot 00$ \\
\hline $18: 2 n-6$ & $19 \cdot 11$ & $19 \cdot 22$ & $19 \cdot 15$ & 19.01 \\
\hline $18: 3 n-6$ & 0.33 & 0.60 & 0.40 & 0.41 \\
\hline $20: 4 n-6$ & 0.32 & $5 \cdot 20$ & 0.46 & $2 \cdot 88$ \\
\hline $22: 4 n-6$ & 0.32 & 0.30 & 0.06 & 0.12 \\
\hline n-6 PUFA & $20 \cdot 08$ & $25 \cdot 32$ & $20 \cdot 08$ & 22.43 \\
\hline $18: 3 n-3$ & $16 \cdot 01$ & 17.40 & $17 \cdot 17$ & $17 \cdot 36$ \\
\hline $20: 5 n-3$ & 0.32 & 0.39 & $5 \cdot 18$ & 2.62 \\
\hline $22: 6 n-3$ & 0.41 & 0.28 & 0.11 & 0.12 \\
\hline$n-3$ PUFA & $16 \cdot 75$ & 18.07 & 22.46 & $20 \cdot 11$ \\
\hline PUFA & 36.83 & 43.39 & 42.54 & 42.54 \\
\hline$n-6: n-3$ & 1.20 & 1.40 & 0.89 & $1 \cdot 12$ \\
\hline ARA:EPA & 1.00 & 13.39 & 0.09 & $1 \cdot 10$ \\
\hline
\end{tabular}

ARA, arachidonic acid.

During the feeding experiment, water was circularly filtered and renewed by $1 / 5$ daily to maintain acceptable water quality. Water temperature was controlled at $28^{\circ} \mathrm{C}$. The dissolved $\mathrm{O}_{2}$ was approximately at saturation $(9 \mathrm{mg} / \mathrm{l})$ through continuous aeration. The photoperiod was $12 \mathrm{~h}$ light-12h dark (from 08.00 to 20.00 hours). Dead fish were weighed and recorded

\section{Sampling procedure}

After 10 weeks of feeding, the fish were fasted for $24 \mathrm{~h}$. All of the fish were anaesthetised with tricaine methanesulfonate (MS222; $0.06 \mathrm{~g} / \mathrm{l}$ ). Fish were weighed and their body lengths were measured. Six fish per aquarium were randomly selected for blood collection from the caudal vein, and sampled blood was allowed to clot at $4^{\circ} \mathrm{C}$ for $6 \mathrm{~h}$. Serum samples were collected after centrifugation of the clot for $10 \mathrm{~min}\left(825 \mathrm{~g}, 4^{\circ} \mathrm{C}\right)$, and then they were pooled two by two in each aquarium, frozen in liquid $\mathrm{N}_{2}$ and stored at $-80^{\circ} \mathrm{C}$ until analysis. The remaining fish were killed and dissected. After weighing the total visceral weight, the hepatopancreas, intraperitoneal fat (IPF), kidney, spleen and intestine were stripped and weighed. Afterwards, samples of hepatopancreas and IPF from three fish/ aquarium were stored at $-20^{\circ} \mathrm{C}$ for fatty acid composition analyses; those from another six fish/aquarium were frozen in liquid $\mathrm{N}_{2}$ and then stored at $-80^{\circ} \mathrm{C}$ for gene expression or enzyme activities measurements; and those from the other three fish/aquarium were fixed in paraformaldehyde solution for histology analysis. All experimental animal procedures were approved by the institutional animal care and use committee and performed in accordance with national and institutional regulations on the care and use of experimental animals.

Specific growth rate (SGR), feed conversion ratio (FCR), hepatopancreas index (HI), intraperitoneal fat index (IPFI), relative kidney weight (RKW), relative spleen weight (RSW), relative intestine weight (RIW), viscera index (VI), condition factor (CF) and survival rate (SR) were calculated using the following formulae:

$$
\begin{aligned}
& \text { SGR }=(\text { Ln final weight }- \text { Ln initial weight }) \times 100 / 70 d \\
& \text { FCR }=\text { amount of feed given } / \text { weight gain }(\mathrm{g}) \\
& \text { HI }(\%)=\text { hepatopancreas weight } \times 100 / \text { body weight } \\
& \text { IPFI }(\%)=\text { IPF weight } \times 100 / \text { body weight } \\
& \text { RKW }(\%)=\text { kidney weight } \times 100 / \text { body weight } \\
& \text { RSW }(\%)=\text { spleen weight } \times 100 / \text { body weight } \\
& \text { RIW }(\%)=\text { intestine weight } \times 100 / \text { body weight } \\
& \text { VI }(\%)=\text { viscera weight } \times 100 / \text { body weight } \\
& \text { CF }\left(g / \mathrm{cm}^{3}\right)=\text { body weight } \times 100 / \text { body length }{ }^{3} \\
& \text { SR }(\%)=\text { final number of fish } \times 100 / \text { initial number of fish } .
\end{aligned}
$$

\section{Proximate composition analysis}

The proximate compositions of the diets were determined according to the methods of Association of Official Analytical Chemists Procedures $(1995)^{(41)}$. In brief, samples were dried to a constant weight to determine moisture at $105^{\circ} \mathrm{C}$. Crude protein was determined by measuring $\mathrm{N}(\mathrm{N} \times 6.25)$ of the samples using the Kjeldahl method. Crude lipid was measured by diethyl ether extraction using the Soxhlet method. Crude ash was determined by combustion at $550^{\circ} \mathrm{C}$ in a muffle furnace.

\section{Fatty acid composition analysis}

Lipid extraction of tissues (hepatopancreas and IPF; 3 individuals/aquarium) and diets was performed by using chloroformmethanol $(2: 1, \mathrm{v} / \mathrm{v})$ based on the method of Folch et al. $^{(42)}$. Extracts were dried under vacuum, and then the tissue lipids were dissolved in chloroform and separated into polar lipids (PL) and non-polar lipids (NPL) using Silica Cartridges according to the method of Juaneda \& Rocquelin ${ }^{(43)}$. A volume of $20 \mathrm{ml}$ of chloroform was used to elute the NPL fraction and $30 \mathrm{ml}$ of methanol was added to elute the PL fraction. Extracts were then dried under vacuum again. The preparation of fatty acid methyl esters (FAME) was performed based on the method described previously ${ }^{(4,39)}$. In brief, $1 \mathrm{ml}$ of hexane was added to dissolve the lipid fractions, and methyl esterification was performed for $1 \mathrm{~h}$ after adding $1 \mathrm{ml}$ of potassium hydroxide methanol $(0.4 \mathrm{M})$. Then, $2 \mathrm{ml}$ of distilled water was added to separate the mixture into two layers. The upper layer was separated and used for GC analysis. The FAME were determined by an Agilent 7820a Series GC (Agilent Technologies) equipped with a flame ionisation detector and capillary column (HP-88, length $100 \mathrm{~m}$, internal diameter $0.25 \mathrm{~mm}$, film thickness $0.20 \mu \mathrm{m}$; Agilent Technologies). Individual methyl esters were identified through comparison with known standards (47015-U; Sigma-Aldrich, Inc.). The results of identified fatty acids were presented as percentage of total fatty acids. 
Table 3. Primers used in real-time quantitative PCR

\begin{tabular}{llll}
\hline Target genes & Accession no. & Forward (5'-3') & Reverse (5'-3') \\
\hline PPAR & EU847421 & GCATCTGTACGAGTCCTATCT & GAGACTTCATGTCGTGGATAAC \\
CCAAT enhancer-binding protein a & KR071139 & ACCCACATACCACCACTCTCAACA & TTTCCCTCGATCGCCCATCTTCAT \\
Diacylglycerol O-acyltransferase & KY800895 & CACCTTCCAAGTACCTTCTG & AGATCCCACTCGCCTATT \\
ApoE & KY800896 & CTTAAGAGCTCCACGCTTATC & GTGTAGTAGGACGCACATTTAT \\
Lipoprotein lipase & FJ716100 & TACAGCGGCGTTCACACTTG & CTACATGAGCACCAAGACTG \\
Fatty acid synthase & GQ466046 & CCTCAGCTTACAGCAGAATC & CTCTTCAGCAAGGGAGTTAG \\
$\begin{array}{l}\text { PPARa } \\
\text { Adipose TAG lipase }\end{array}$ & FJ623265 & CGCTGAGGTTCGGATATTT & ACGTCACCTGGTCATTAAG \\
Hormone-sensitive lipase & HQ845211 & TCGTGCAAGCGTGTATATG & GCTCGTACTGAGGCAATTA \\
Carnitine palmitoyltransferase 1 & HQ446238 & TGGAACGTTACTGAGTCTGG & AAGCGCACGTTGACTGG \\
B-Actin & JF728839 & GCATCCATGACACGTTATTC & GAAGTTCTCTTCTCTCGTCTC \\
\hline & DO211096 & TCCACCTTCCAGCAGATGTGGATT & AGTTTGAGTCGGCGTGAAGTGGTA
\end{tabular}

\section{Antioxidant status and lysozyme activity}

Serum SOD activity, CAT activity, GSH-Px activity, malondialdehyde (MDA) content and the activity of lysozyme (LZM) were measured with the use of assay kits (Jiancheng Biotech Co.).

\section{Histological processing and morphological evaluation}

The samples of fixed hepatopancreas and IPF were washed in tap water for $12 \mathrm{~h}$, followed by a routine dehydration in a graded series of ethanol (30, 50, 70, 80, 90, 95 and 100\% twice). The samples were then equilibrated in xylene and embedded in paraffin based on the standard histological techniques, as described previously ${ }^{(44)}$. Afterwards, sections were cut at $5 \mu \mathrm{m}$ with the use of a rotary microtome (RM2235; Leica) and mounted in glass slides, which were then stained with haematoxylin-eosin. Histological samples were observed and photographed by using an upright microscopy (Leica Biosystems). The average adipocyte size per image was quantified using Photoshop (Adobe), as previously described by Osman et $a l .{ }^{(45)}$. An average value across five non-overlapping images (five/section) was calculated for each group.

\section{NEFA and TAG assays}

NEFA from serum and hepatopancreas and the TAG content from hepatopancreas were measured by using the NEFA assay kit or TAG assay kit (Jiancheng Biotech Co.). These two indexes were measured based on the user's manual step by step. All assays were performed in triplicate in each aquarium.

\section{Hepatopancreatic lipid metabolism enzyme activities}

Lipid-metabolism-related enzymes, including the lipoprotein lipase (LPL), hepatic lipase (HL) and malate dehydrogenase $(\mathrm{MDH})$, in the hepatopancreas were measured by using the assay kits (Jiancheng Biotech Co.) according to the method in the specification.

\section{Real-time quantitative RT-PCR}

Hepatopancreas and IPF from three fish per aquarium were used for the detection of gene expression. RNA extraction, complementary DNA synthesis and gene expression measurements were performed as described previously ${ }^{(4)}$. The primer sequences for $\beta$-actin, $L P L$, fatty acid synthase $(F A S)$, diacylglycerol $O$-acyltransferase (DGAT), apoE, $P P A R \alpha$, adipose TAG lipase $(A T G L), P P A R \gamma$, CCAAT enhancer-binding protein $\alpha(C / E B P \alpha)$, hormone-sensitive lipase $(H S L)$ and carnitine palmitoyltransferase $1(C P T-1)$ are listed in Table 3. After the PCR reaction, melting curves were analysed to confirm that single products were obtained in these reactions. A relative quantification method, the comparative CT method $\left(2^{-\Delta \Delta C_{t}}\right)$, was used to calculate the gene expression values, as described in the literature ${ }^{(46,47)}$.

\section{Statistical analysis}

All data were expressed as means and standard deviations. Percentage data were arcsine-transformed before analysis. One-way ANOVA was used to compare differences among the experimental groups, followed by Duncan's post hoc tests. All analyses, including the principle component analysis (PCA) of the fatty acids, were conducted using PASW Statistics 18 (SPSS). A significance level of $P<0.05$ was used for all tests.

\section{Results \\ Growth performance and biological parameters}

The growth of fish doubled compared with the initial weight (Table 4). Fish fed 0.30\% EPA exhibited significantly higher final weight and SGR in comparison with fish fed the control diet $(P<0.05)$. HI in the ARA, EPA and ARA + EPA groups presented significantly lower values than those in the control group $(P<0.05)$. The IPFI value was inhibited in the ARA group compared with the control $(P<0.05)$. Interestingly, fish subjected to ARA + EPA had higher RSW than those subjected to control $(P<0.05)$. No statistically significant difference was found in other indices such as FCR, RKW, RIW, VI, CF and SR $(P>0.05)$.

\section{Fatty acid composition}

Lipids in the hepatopancreas and IPF were separated for NPL and PL fractions, and then fatty acid compositions were analysed (Tables 5 and 6). ARA and EPA presented higher levels in the PL $(3.56-5.86 \%$ for ARA; $0.40-1.46 \%$ for EPA) than NPL $(0.41-0.98 \%$ for ARA; $0 \cdot 25-0.50 \%$ for EPA) of the 
Table 4. Effects of dietary arachidonic acid (ARA) and EPA on the growth performance, feed utilisation, survival and biological parameters of juvenile grass carp

(Mean values and standard deviations; $n$ 3/group)

\begin{tabular}{|c|c|c|c|c|c|c|c|c|}
\hline & \multicolumn{8}{|c|}{ Groups } \\
\hline & \multicolumn{2}{|c|}{ Control } & \multicolumn{2}{|c|}{ ARA } & \multicolumn{2}{|c|}{ EPA } & \multicolumn{2}{|c|}{$A R A+E P A$} \\
\hline & Mean & SD & Mean & SD & Mean & SD & Mean & SD \\
\hline Initial weight (g) & $10 \cdot 16$ & 0.14 & $10 \cdot 18$ & $0 \cdot 12$ & $10 \cdot 25$ & 0.10 & $10 \cdot 25$ & 0.33 \\
\hline Final weight $(g)$ & $28 \cdot 12^{b}$ & 2.35 & $29 \cdot 13^{\mathrm{b}}$ & 0.80 & $32 \cdot 30^{\mathrm{a}}$ & 1.79 & $29 \cdot 74^{a, b}$ & 0.90 \\
\hline Specific growth rate & $1.45^{\mathrm{b}}$ & 0.13 & $1.50^{\mathrm{a}, \mathrm{b}}$ & 0.06 & $1.64^{\mathrm{a}}$ & 0.07 & $1.52^{\mathrm{a}, \mathrm{b}}$ & 0.05 \\
\hline Feed conversion ratio & 1.59 & 0.06 & 1.40 & 0.09 & 1.21 & 0.03 & 1.37 & 0.02 \\
\hline Hepatopancreas index (\%) & $3.35^{a}$ & 0.08 & $2 \cdot 78^{\mathrm{b}}$ & 0.18 & $3.00^{\mathrm{b}}$ & 0.11 & $2 \cdot 96^{\mathrm{b}}$ & 0.24 \\
\hline Intraperitoneal fat index (\%) & $1.64^{\mathrm{a}}$ & 0.07 & $1.33^{\mathrm{b}}$ & 0.16 & $1.56^{a, b}$ & 0.23 & $1.51^{\mathrm{a}, \mathrm{b}}$ & 0.05 \\
\hline Relative kidney weight (\%) & 0.38 & 0.02 & 0.40 & 0.03 & 0.38 & 0.02 & 0.44 & 0.05 \\
\hline Relative spleen weight (\%) & $0.15^{\mathrm{b}}$ & 0.01 & $0 \cdot 16^{\mathrm{a}, \mathrm{b}}$ & 0.02 & $0 \cdot 15^{\mathrm{a}, \mathrm{b}}$ & 0.02 & $0.18^{\mathrm{a}}$ & 0.02 \\
\hline Relative intestine weight (\%) & 3.32 & 0.22 & 3.54 & 0.67 & 3.66 & 0.59 & 3.80 & 0.54 \\
\hline Visceral index (\%) & $16 \cdot 07$ & 0.53 & $14 \cdot 81$ & 1.34 & $15 \cdot 64$ & 0.91 & $15 \cdot 44$ & 1.38 \\
\hline Condition factor $\left(\mathrm{g} / \mathrm{cm}^{3}\right)$ & 1.99 & 0.02 & 1.98 & 0.03 & 2.02 & 0.04 & 2.01 & 0.03 \\
\hline Survival rate $(\%)$ & $96 \cdot 30$ & $3 \cdot 21$ & $98 \cdot 15$ & $3 \cdot 21$ & 100 & 0.00 & $98 \cdot 15$ & 3.21 \\
\hline
\end{tabular}

${ }^{\mathrm{a}, \mathrm{b}}$ Mean values within a row with unlike superscript letters were significantly different $(P<0.05)$.

Table 5. Effects of dietary arachidonic acid (ARA) and EPA on the fatty acid composition (percentage of total fatty acids) in the non-polar lipid (NPL) and polar lipid (PL) fraction of hepatopancreas in juvenile grass carp

(Mean values and standard deviations; $n$ 3/group)

\begin{tabular}{|c|c|c|c|c|c|c|c|c|c|c|c|c|c|c|c|c|}
\hline \multirow[b]{3}{*}{ Fatty acids } & \multicolumn{8}{|c|}{ NPL } & \multicolumn{8}{|c|}{$\mathrm{PL}$} \\
\hline & \multicolumn{2}{|c|}{ Control } & \multicolumn{2}{|c|}{ ARA } & \multicolumn{2}{|c|}{ EPA } & \multicolumn{2}{|c|}{$\mathrm{ARA}+\mathrm{EPA}$} & \multicolumn{2}{|c|}{ Control } & \multicolumn{2}{|c|}{ ARA } & \multicolumn{2}{|c|}{ EPA } & \multicolumn{2}{|c|}{$A R A+E P A$} \\
\hline & Mean & SD & Mean & SD & Mean & SD & Mean & SD & Mean & SD & Mean & SD & Mean & SD & Mean & SD \\
\hline $14: 0$ & $2 \cdot 70^{\mathrm{a}}$ & 0.08 & $2 \cdot 41^{\mathrm{b}}$ & 0.05 & $2 \cdot 48^{\mathrm{b}}$ & 0.08 & $2 \cdot 61^{a, b}$ & 0.16 & $2 \cdot 64$ & 0.25 & $2 \cdot 36$ & 0.17 & $2 \cdot 33$ & 0.24 & 2.43 & 0.31 \\
\hline $16: 0$ & $25 \cdot 34$ & 1.32 & 24.33 & 1.83 & $25 \cdot 33$ & 0.94 & $24 \cdot 12$ & $2 \cdot 52$ & 31.98 & 1.21 & $31 \cdot 12$ & 5.44 & 28.44 & 1.32 & 28.63 & 3.37 \\
\hline $18: 0$ & $9 \cdot 03^{\mathrm{a}}$ & 0.53 & $8.46^{a}$ & 0.54 & $8 \cdot 34^{a}$ & 0.28 & $7.45^{\mathrm{b}}$ & 0.24 & 21.63 & 1.51 & $21 \cdot 79$ & $5 \cdot 13$ & $18 \cdot 32$ & 3.01 & 17.60 & 2.42 \\
\hline SFA & 37.07 & 1.93 & $35 \cdot 21$ & $2 \cdot 33$ & $36 \cdot 15$ & 1.27 & $34 \cdot 18$ & 2.90 & $56 \cdot 26$ & 2.93 & $55 \cdot 27$ & 10.67 & 49.09 & 3.91 & 48.67 & 5.25 \\
\hline $16: 1 n-7$ & 8.28 & 0.23 & 8.38 & 0.29 & 8.27 & 0.37 & 8.65 & 0.60 & $5 \cdot 60$ & 0.67 & $5 \cdot 31$ & 1.86 & $6 \cdot 32$ & $1 \cdot 14$ & $6 \cdot 31$ & 1.08 \\
\hline $18: 1 n-9$ & $45 \cdot 78$ & 1.31 & $47 \cdot 01$ & 0.74 & 46.46 & 1.48 & 47.48 & 0.85 & $22 \cdot 40$ & 3.79 & $21 \cdot 84$ & $8 \cdot 34$ & $26 \cdot 29$ & $4 \cdot 18$ & $25 \cdot 35$ & 2.58 \\
\hline $20: 1 n-9$ & 0.59 & 0.16 & 0.54 & 0.08 & 0.41 & 0.15 & 0.45 & 0.03 & $1 \cdot 23^{\mathrm{a}}$ & 0.05 & $0.93^{a, b}$ & 0.27 & $0.97^{a, b}$ & 0.16 & $0.74^{\mathrm{b}}$ & 0.14 \\
\hline MUFA & 54.64 & 1.19 & 55.94 & 0.87 & $55 \cdot 14$ & 1.65 & 56.59 & 0.37 & $29 \cdot 23$ & 4.44 & 28.08 & 9.97 & 33.59 & $5 \cdot 17$ & $32 \cdot 41$ & 3.45 \\
\hline $18: 2 n-6$ & 3.85 & 1.28 & 3.88 & 1.32 & 3.64 & 0.78 & 4.56 & 1.91 & 3.14 & 0.58 & $3 \cdot 17$ & $1 \cdot 10$ & $3 \cdot 27$ & 0.14 & 3.83 & 1.66 \\
\hline $18: 3 n-6$ & 0.20 & 0.02 & 0.21 & 0.06 & 0.15 & 0.02 & 0.22 & 0.10 & 0.19 & 0.05 & 0.15 & 0.05 & 0.14 & 0.08 & 0.13 & 0.04 \\
\hline $20: 4 n-6$ & $0.45^{\mathrm{b}}$ & 0.14 & $0.98^{\mathrm{a}}$ & 0.33 & $0.41^{b}$ & 0.15 & $0.71^{a, b}$ & 0.21 & $3.67^{c}$ & 0.44 & $5 \cdot 86^{a}$ & 0.17 & $3.56^{\mathrm{C}}$ & 0.59 & $4.89^{b}$ & 0.36 \\
\hline $22: 4 n-6$ & 0.16 & 0.06 & 0.25 & 0.20 & 0.26 & 0.23 & 0.12 & 0.07 & 0.13 & 0.09 & 0.52 & 0.33 & 0.41 & 0.20 & 0.44 & 0.13 \\
\hline$n-6$ PUFA & 4.66 & 1.41 & $5 \cdot 33$ & 1.90 & 4.46 & 1.14 & $5 \cdot 61$ & $2 \cdot 26$ & $7 \cdot 13^{b}$ & 0.84 & $9 \cdot 70^{a}$ & 0.75 & $7 \cdot 38^{a, b}$ & 0.69 & $9 \cdot 30^{a, b}$ & 2.05 \\
\hline $18: 3 n-3$ & 1.20 & 0.79 & 1.22 & 0.38 & 1.26 & 0.31 & 1.63 & 0.94 & 0.71 & 0.39 & 0.67 & 0.44 & 1.48 & 1.32 & 0.83 & 0.12 \\
\hline $18: 4 n-3$ & $1 \cdot 70$ & 0.30 & 1.73 & 0.28 & 1.67 & 0.31 & $1 \cdot 17$ & 0.78 & 0.64 & $0 \cdot 10$ & 0.66 & 0.29 & 0.84 & 0.10 & 0.66 & 0.09 \\
\hline $20: 5 n-3$ & 0.34 & 0.06 & 0.25 & $0 \cdot 18$ & 0.50 & 0.17 & 0.35 & 0.13 & $0.52^{\mathrm{c}}$ & 0.14 & $0.40^{c}$ & 0.23 & $1.46^{\mathrm{a}}$ & 0.03 & $0.93^{\mathrm{b}}$ & 0.20 \\
\hline $22: 5 n-3$ & 0.07 & 0.05 & 0.08 & 0.03 & 0.27 & 0.29 & 0.08 & 0.02 & $0.19^{d}$ & 0.04 & $0.27^{c}$ & 0.03 & $0.45^{\mathrm{a}}$ & 0.05 & $0.36^{b}$ & 0.02 \\
\hline $22: 6 n-3$ & 0.32 & 0.15 & 0.25 & 0.04 & 0.56 & 0.36 & 0.38 & 0.07 & 5.32 & 0.95 & 4.96 & 0.24 & 6.35 & 0.87 & $6 \cdot 20$ & 0.56 \\
\hline$n-3$ PUFA & 3.63 & 1.14 & 3.53 & 0.80 & $4 \cdot 26$ & 1.28 & 3.62 & 0.37 & $7.38^{b}$ & 1.06 & $6.95^{\mathrm{b}}$ & 0.31 & $9.94^{\mathrm{a}}$ & 0.95 & $9 \cdot 62^{\mathrm{a}}$ & 0.94 \\
\hline PUFA & 8.29 & 2.55 & 8.86 & 2.69 & $8 \cdot 71$ & 2.40 & 9.23 & 2.62 & $14.51^{\mathrm{b}}$ & 1.86 & $16 \cdot 65^{a, b}$ & 1.01 & $17 \cdot 32^{\mathrm{a}, \mathrm{b}}$ & 1.64 & $18 \cdot 93^{a}$ & 2.90 \\
\hline$n-6: n-3$ & 1.29 & 0.04 & 1.48 & 0.19 & 1.06 & 0.07 & 1.52 & 0.45 & $0.97^{\mathrm{b}}$ & 0.06 & $1.39^{\mathrm{a}}$ & 0.07 & $0.74^{\mathrm{C}}$ & 0.00 & $0.96^{\mathrm{b}}$ & 0.13 \\
\hline ARA:EPA & $1.31^{b}$ & 0.23 & $5.06^{\mathrm{a}}$ & 3.13 & $0.81^{\mathrm{b}}$ & 0.04 & $2 \cdot 14^{\mathrm{a}, \mathrm{b}}$ & 072 & $7 \cdot 62^{b}$ & 3.10 & $18 \cdot 21^{a}$ & 9.19 & $2 \cdot 44^{\mathrm{b}}$ & 0.46 & $5 \cdot 49^{b}$ & 1.47 \\
\hline
\end{tabular}

a,b,c,d Mean values within a row with unlike superscript letters were significantly different $(P<0.05)$.

hepatopancreas, whereas there was no apparent difference in the IPF $(0.75-2.47 \%$ in NPL and $0.63-1.61 \%$ in PL for ARA; $0 \cdot 47-1 \cdot 30 \%$ in NPL and $0 \cdot 51-1 \cdot 20 \%$ in PL for EPA).

To be detailed, in the hepatopancreas, the highest ARA contents were presented in fish fed ARA both in NPL and PL, followed by ARA + EPA $(P<0 \cdot 05)$. However, significantly different contents of EPA were only found in the PL, which showed significantly higher level in the EPA group, followed by the ARA+EPA group $(P<0 \cdot 05)$. Moreover, EPA feeding fish also showed increased $22: 5 n-3$ content in the PL compared with the control $(P<0.05)$. These resulted in the increase of the total $n$-3 PUFA. ARA:EPA ratio exhibited a similar trend with those in the diets $(P<0.05)$ (Table 5 ).

In the IPF, dietary ARA significantly increased the ARA proportion both in NPL and PL $(P<0.05)$; paralleled trends also applied to $22: 4 n-6$ in the NPL and PL, and 18:3n-6 in the NPL $(P<0 \cdot 05)$. Similarly, EPA content increased in fish fed EPA and ARA + EPA in these two lipid fractions $(P<0 \cdot 05)$. Moreover, dietary EPA also 
Table 6. Effects of dietary arachidonic acid (ARA) and EPA on the fatty acid composition (percentage of total fatty acids) in the non-polar lipid (NPL) and polar lipid (PL) fraction of intraperitoneal fat in juvenile grass carp

(Mean values and standard deviations; $n$ 3/group)

\begin{tabular}{|c|c|c|c|c|c|c|c|c|c|c|c|c|c|c|c|c|}
\hline \multirow[b]{3}{*}{ Fatty acids } & \multicolumn{8}{|c|}{ NPL } & \multicolumn{8}{|c|}{ PL } \\
\hline & \multicolumn{2}{|c|}{ Control } & \multicolumn{2}{|c|}{ ARA } & \multicolumn{2}{|c|}{ EPA } & \multicolumn{2}{|c|}{$A R A+E P A$} & \multicolumn{2}{|c|}{ Control } & \multicolumn{2}{|c|}{ ARA } & \multicolumn{2}{|c|}{ EPA } & \multicolumn{2}{|c|}{$A R A+E P A$} \\
\hline & Mean & SD & Mean & SD & Mean & SD & Mean & SD & Mean & SD & Mean & SD & Mean & SD & Mean & SD \\
\hline $14: 0$ & $2 \cdot 35$ & 0.05 & $2 \cdot 15$ & 0.08 & $2 \cdot 15$ & 0.17 & $2 \cdot 28$ & 0.12 & 3.43 & 0.08 & 2.95 & 0.21 & 3.07 & 0.52 & 3.04 & 0.33 \\
\hline $16: 0$ & $20 \cdot 81$ & 1.34 & 19.88 & $1 \cdot 11$ & 20.49 & 1.14 & 20.02 & 0.79 & $40 \cdot 36$ & 2.95 & 35.52 & 8.95 & $30 \cdot 10$ & 4.40 & 30.57 & 4.19 \\
\hline $18: 0$ & $4 \cdot 71$ & 0.25 & $4 \cdot 70$ & 0.29 & 4.46 & 0.56 & 4.45 & 0.25 & $17 \cdot 40^{\mathrm{a}}$ & 1.46 & $13 \cdot 61^{a, b}$ & 4.40 & $10 \cdot 06^{b}$ & 0.56 & $11 \cdot 14^{\mathrm{b}}$ & 0.38 \\
\hline SFA & 28.04 & 1.48 & $26 \cdot 89$ & 1.38 & $27 \cdot 26$ & 1.88 & 26.93 & 1.08 & $61 \cdot 19^{a}$ & 3.00 & $52 \cdot 08^{\mathrm{a}, \mathrm{b}}$ & 13.53 & $43 \cdot 23^{b}$ & 4.52 & $44.75^{b}$ & 4.89 \\
\hline $16: 1 n-7$ & 7.52 & 0.67 & 6.62 & 0.50 & 7.49 & 0.36 & 7.09 & 0.97 & $4 \cdot 51^{b}$ & 0.17 & $4.56^{\mathrm{b}}$ & 0.59 & $6 \cdot 37^{\mathrm{a}}$ & 0.61 & $5 \cdot 66^{\mathrm{a}}$ & 0.74 \\
\hline $18: 1 n-9$ & 40.58 & 0.93 & 39.09 & 1.01 & 40.57 & 1.24 & 39.06 & 1.37 & $19 \cdot 89^{b}$ & 0.88 & $23 \cdot 80^{a, b}$ & 5.95 & $28 \cdot 36^{a}$ & 1.99 & $26 \cdot 18^{a, b}$ & 2.68 \\
\hline $20: 1 n-9$ & $0.41^{a}$ & 0.02 & $0.36^{a, b}$ & 0.05 & $0.33^{b}$ & 0.03 & $0.36^{a, b}$ & 0.04 & 0.22 & 0.06 & 0.26 & 0.06 & 0.29 & 0.17 & 0.22 & 0.10 \\
\hline MUFA & 48.51 & 1.56 & $46 \cdot 07$ & 1.48 & $48 \cdot 39$ & 1.56 & 46.52 & $2 \cdot 34$ & $24.62^{b}$ & 1.03 & $28.63^{a, b}$ & 6.51 & $35 \cdot 01^{a}$ & $2 \cdot 35$ & $32 \cdot 07^{a, b}$ & 3.49 \\
\hline $18: 2 n-6$ & 12.59 & 1.72 & 13.42 & 1.23 & 11.72 & 0.33 & 13.07 & 1.42 & 7.48 & 1.08 & 9.42 & 3.88 & $10 \cdot 59$ & 0.94 & $10 \cdot 55$ & 0.84 \\
\hline $18: 3 n-6$ & $0.37^{b}$ & 0.06 & $0.45^{\mathrm{a}}$ & 0.03 & $0.28^{\mathrm{C}}$ & 0.01 & $0.36^{\mathrm{b}}$ & 0.04 & 0.27 & 0.05 & 0.36 & 0.08 & 0.30 & 0.08 & 0.27 & 0.00 \\
\hline $20: 4 n-6$ & $0.75^{c}$ & 0.04 & $2 \cdot 47^{\mathrm{a}}$ & 0.17 & $0.80^{\mathrm{C}}$ & 0.10 & $1.69^{b}$ & 0.14 & $0.63^{b}$ & 0.13 & $1 \cdot 61^{a}$ & 0.44 & $0.70^{\mathrm{b}}$ & 0.25 & $1.32^{\mathrm{a}}$ & 0.28 \\
\hline $22: 4 n-6$ & $0 \cdot 19^{b}$ & 0.04 & $0.42^{\mathrm{a}}$ & 0.03 & $0.27^{a, b}$ & 0.06 & $0.32^{a, b}$ & 0.18 & $0.26^{\mathrm{b}}$ & 0.13 & $0.96^{\mathrm{a}}$ & 0.29 & $0.39^{b}$ & 0.21 & $0.63^{a, b}$ & 0.08 \\
\hline$n-6$ PUFA & $13.90^{b}$ & 1.78 & $16 \cdot 77^{\mathrm{a}}$ & 1.43 & $13 \cdot 06^{\mathrm{b}}$ & 0.31 & $15 \cdot 43^{a, b}$ & 1.69 & 8.65 & 1.15 & 12.01 & 4.33 & 11.97 & 1.40 & $13 \cdot 10$ & 1.07 \\
\hline $18: 3 n-3$ & 7.79 & 0.70 & 8.52 & 0.71 & 7.91 & 0.10 & $8 \cdot 38$ & 0.57 & $3.99^{b}$ & 0.48 & $5 \cdot 69^{a, b}$ & 2.49 & $6 \cdot 85^{\mathrm{a}}$ & 0.31 & $6 \cdot 96^{\mathrm{a}}$ & 0.37 \\
\hline $18: 4 n-3$ & ND & & ND & & ND & & ND & & ND & & ND & & ND & & ND & \\
\hline $20: 5 n-3$ & $0.47^{c}$ & 0.06 & $0.47^{c}$ & 0.04 & $1.30^{\mathrm{a}}$ & 0.04 & $0.81^{b}$ & $0 \cdot 10$ & $0.51^{\mathrm{b}}$ & 0.26 & $0.53^{b}$ & 0.13 & $1 \cdot 20^{\mathrm{a}}$ & 0.27 & $0.94^{a}$ & 0.18 \\
\hline $22: 5 n-3$ & $0.36^{c}$ & 0.07 & $0.35^{c}$ & 0.01 & $0.69^{a}$ & 0.04 & $0.57^{\mathrm{b}}$ & 0.06 & 0.28 & 0.37 & 0.29 & 0.12 & 0.69 & 0.24 & 0.53 & 0.11 \\
\hline $22: 6 n-3$ & $0.93^{\mathrm{b}}$ & 0.07 & $0.93^{\mathrm{b}}$ & 0.02 & $1.38^{\mathrm{a}}$ & 0.09 & $1.36^{\mathrm{a}}$ & $0 \cdot 10$ & $0.76^{\mathrm{b}}$ & 0.27 & $0.77^{\mathrm{b}}$ & 0.18 & $1.50^{\mathrm{a}}$ & 0.49 & $1 \cdot 21^{a, b}$ & 0.29 \\
\hline$n-3$ PUFA & $9.56^{\mathrm{b}}$ & 0.90 & $10 \cdot 27^{a, b}$ & 0.76 & $11 \cdot 28^{\mathrm{a}}$ & 0.11 & $11 \cdot 12^{\mathrm{a}}$ & 0.74 & $5 \cdot 54^{\mathrm{b}}$ & 1.14 & $7 \cdot 28^{a, b}$ & $2 \cdot 74$ & $10 \cdot 08^{\mathrm{a}}$ & $1 \cdot 11$ & $9.79^{\mathrm{a}}$ & 0.84 \\
\hline PUFA & 23.45 & 2.67 & 27.04 & $2 \cdot 19$ & $24 \cdot 34$ & 0.34 & $26 \cdot 55$ & 2.43 & $14 \cdot 19^{b}$ & 2.04 & $19 \cdot 29^{a, b}$ & 7.06 & $21 \cdot 76^{a, b}$ & $2 \cdot 19$ & $23 \cdot 18^{\mathrm{a}}$ & $2 \cdot 15$ \\
\hline$n-6: n-3$ & $1.45^{\mathrm{b}}$ & 0.06 & $1.63^{\mathrm{a}}$ & 0.02 & $1 \cdot 16^{\mathrm{c}}$ & 0.03 & $1.38^{\mathrm{b}}$ & 0.06 & $1.59^{\mathrm{a}}$ & 0.23 & $1.66^{\mathrm{a}}$ & 0.05 & $1.22^{\mathrm{b}}$ & 0.07 & $1 \cdot 30^{\mathrm{b}}$ & 0.06 \\
\hline ARA:EPA & $1.59^{c}$ & 0.14 & $5 \cdot 24^{a}$ & 0.20 & $0.61^{d}$ & 0.07 & $2.09^{b}$ & 0.21 & $1.37^{\mathrm{b}}$ & 0.40 & $3 \cdot 22^{\mathrm{a}}$ & 1.21 & $0.58^{\mathrm{b}}$ & 0.14 & $1.41^{\mathrm{b}}$ & 0.05 \\
\hline
\end{tabular}

a,b,c,d Mean values within a row with unlike superscript letters were significantly different $(P<0.05)$.

significantly increased $18: 3 n-3$ in the PL, $22: 5 n-3$ in the NPL, as well as the DHA content both in the NPL and PL fractions $(P<0.05)$. Interestingly, SFA proportion decreased and MUFA proportion increased in the PL of fish fed EPA $(P<0 \cdot 05)$ (Table 6$)$.

The PCA demonstrated different patterns of fatty acid distribution in NPL and PL of these two tissues (Fig. 1). Overall, there exists more distinct difference of the fatty acid composition between these two fractions in the hepatopancreas than those in the adipose tissue. Specifically, in the first component of score plot (Fig. 1(a)), all the hepatopancreas-PL samples are located to the right of the diagram, whereas the adipose tissue NPL are located to the left. The corresponding component plot (Fig. 1(b)) suggests that SFA, ARA and DHA (on the right of the diagram) correspond to the hepatopancreas-PL samples, whereas MUFA and $\mathrm{C}_{18}$ PUFA (on the left right of the diagram), in particular, correspond to the adipose tissue NPL. Furthermore, the second component of the corresponding component plot (Fig. 1(b)) shows that MUFA is located on the negative axis of the second component plot and could correspond to the hepatopancreas NPL samples, whereas the other types of fatty acids could correspond to the adipose tissue PL. Besides, the component plot also demonstrates that adipose tissue is likely to deposit $\mathrm{C}_{18}$ PUFA.

\section{Antioxidant responses and lysozyme activity in serum}

Table 7 shows the status of antioxidant (SOD, CAT and GSH-Px activities and MDA contents) and LZM activity in serum of fish fed different diets. The serum SOD activities and CAT activities were significantly increased in fish fed ARA and EPA diets $(P<0.05)$, whereas fish fed these LC-PUFA showed declined trend of serum GSH-Px activities $(P<0 \cdot 05)$. MDA contents also decreased in fish fed ARA, EPA and ARA + EPA, and significant differences are shown in EPA and ARA + EPA groups $(P<0 \cdot 05)$. As for the serum LZM activities, the treatment groups had higher levels than the control group; EPA and ARA+EPA reached significant levels $(P<0.05)$.

\section{Lipid accumulation}

Fig. 2 shows the phenotype of lipid accumulation of the fish fed various diets. Dietary ARA and EPA treatment groups showed decreased TAG content in the hepatopancreas $(P<0.05)$ (Fig. 2(A) and (C)). On the other hand, in the IPF, adipocyte size in the ARA and ARA + EPA groups presented significantly lower values compared with that in the control $(P<0.05)$ (Fig. 2(B) and $(C)$ ). Interestingly, LC-PUFA treatment groups showed higher serum NEFA levels, and ARA significantly increased the serum NEFA in comparison with the other groups $(P<0.05)$.

\section{Lipid-metabolism-related enzyme activities in hepatopancreas}

The LPL activity of the hepatopancreas was lower in fish fed ARA compared with fish fed control $(P<0.05)$. However, both ARA and EPA groups presented significantly lower HL activities than the ARA + EPA group $(P<0 \cdot 05)$. MDH activities exhibited significantly higher levels in all the LC-PUFA treatment groups compared with those in the control group $(P<0 \cdot 05)$ (Fig. 3). 
(a)

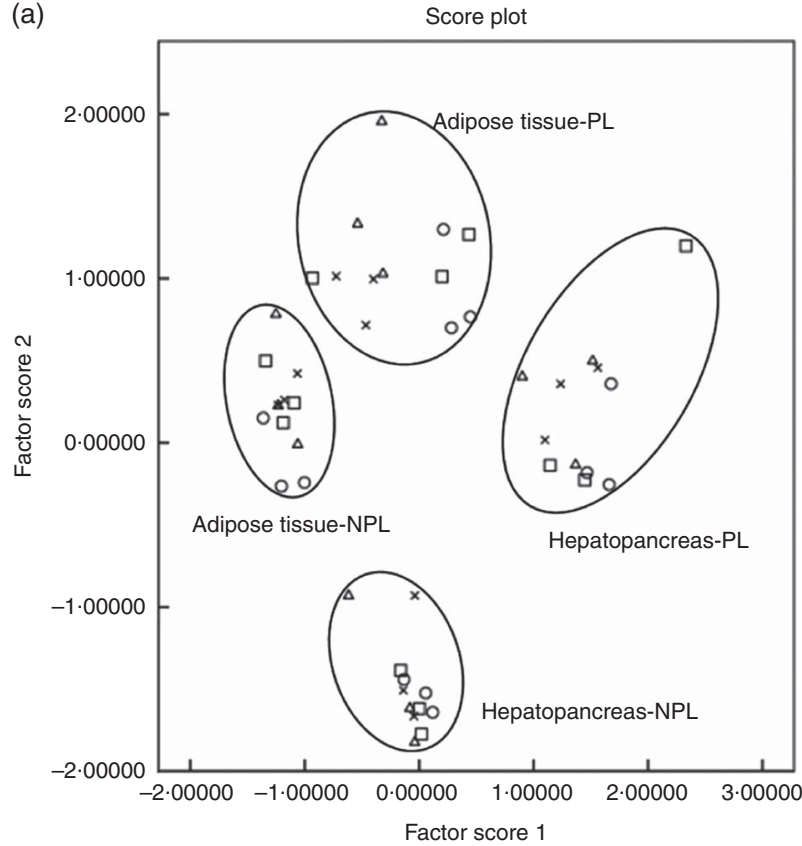

(b)

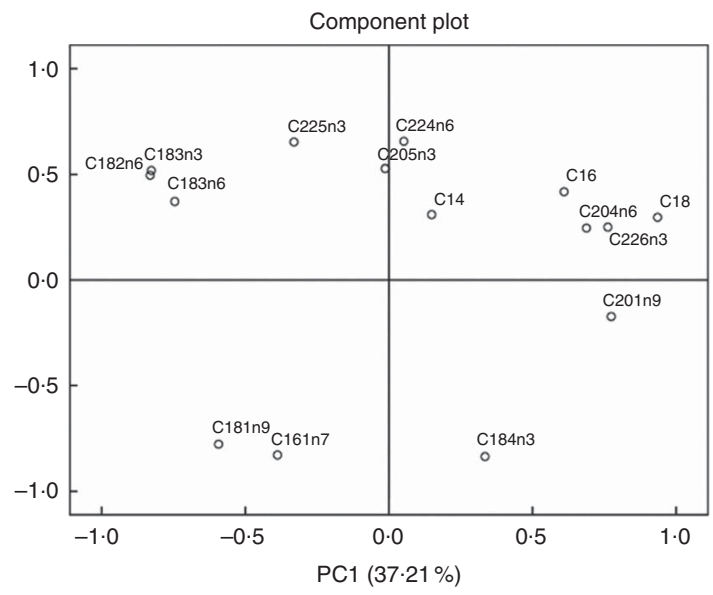

Fig. 1. Score plot (a) and component plot (b) from principal component analysis on fatty acid composition of the non-polar lipid (NPL) and polar lipid (PL) of the hepatopancreas and intraperitoneal fat in juvenile grass carp fed different diets. a: $O$, Control; $\square$, arachidonic acid (ARA); X, EPA; $\triangle$, ARA + EPA.

\section{Lipid-metabolism-related gene expression in hepatopancreas and intraperitoneal fat}

For the hepatopancreas (Fig. 4), dietary ARA, EPA and combined ARA and EPA groups presented significantly lower mRNA expression of FAS, DGAT and apoE $(P<0.05)$. Dietary ARA significantly increased the mRNA expression of $A T G L(P<0.05)$, whereas this gene was decreased in fish subjected to EPA and $\mathrm{ARA}+\mathrm{EPA}$.

In the IPF (Fig. 5), ARA treatment significantly decreased $C / E B P \alpha$ and $L P L$, and dietary ARA and ARA + EPA decreased the expression of FAS $(P<0.05)$. The PPAR $\alpha$ and CPT-1 mRNA level significantly increased in the ARA group compared with the other groups $(P<0.05)$; EPA and ARA + EPA groups also showed a significant increase in these gene levels compared with the controls, whereas they showed lower levels compared with the ARA group. Dietary ARA significantly increased the expression of $A T G L$ and $H S L(P<0.05)$, whereas these genes were not increased under EPA or ARA + EPA treatments.

\section{Discussion}

ARA and EPA exhibit a competitive relationship for binding to some enzymes in fish, such as the eicosanoid synthesis enzymes, phospholipid esterase or fatty acid elongase ${ }^{(6,11)}$. The inverse relationship between EPA and ARA has been reported in fish tissues. Some enzymes have preference for these two fatty acids. ARA is the preferred substrate for eicosanoids, whereas EPA is the preferred substrate for phosphoglycerides ${ }^{(6,11)}$. Moreover, the affinity of the elongase $2 / 5$ is higher for EPA than for $\mathrm{ARA}^{(6,11,14,38)}$. Hence, there must be a complex and competitive regulation of fish physiological processes by these two fatty acids. However, our early studies have suggested a similar function of ARA and $n$ - 3 LC-PUFA in the lipid accumulation, and the mechanism were also analogous ${ }^{(3,4,32,34,36)}$. Thus, in this study, we aimed to compare the effect of dietary ARA and EPA on the performance of grass carp in detail. Our data demonstrated that dietary ARA to some extent has a different role from EPA in controlling growth performance and lipid metabolism of grass carp.

In this study, dietary ARA had no significant effect on the growth performance of grass carp, similar to early studies ${ }^{(4,33)}$. However, a positive effect on the growth was found in fish fed

Table 7. Effects of dietary arachidonic acid (ARA) and EPA on the serum antioxidant responses and lysozyme activity in juvenile grass carp

(Mean values and standard deviations; $n$ 3/group)

\begin{tabular}{|c|c|c|c|c|c|c|c|c|}
\hline & \multicolumn{8}{|c|}{ Groups } \\
\hline & \multicolumn{2}{|c|}{ Control } & \multicolumn{2}{|c|}{ ARA } & \multicolumn{2}{|c|}{ EPA } & \multicolumn{2}{|c|}{$A R A+E P A$} \\
\hline & Mean & SD & Mean & SD & Mean & SD & Mean & SD \\
\hline Superoxide dismutase $(\mathrm{U} / \mu \mathrm{l})$ & $26.45^{\mathrm{b}}$ & 1.55 & $30.90^{\mathrm{a}}$ & 3.38 & $30 \cdot 77^{a}$ & 1.44 & $27 \cdot 85^{a, b}$ & 0.33 \\
\hline Catalase $(\mathrm{U} / \mathrm{ml})$ & $9.09^{b}$ & 1.22 & $13.03^{\mathrm{a}}$ & 1.69 & $12 \cdot 21^{\mathrm{a}}$ & 0.95 & $12 \cdot 47^{\mathrm{a}}$ & 0.61 \\
\hline Glutathion peroxidase $(\mathrm{U} / \mathrm{ml})$ & $748 \cdot 12^{a}$ & $23 \cdot 38$ & $685 \cdot 73^{\mathrm{b}}$ & 19.83 & $630 \cdot 29^{c}$ & $12 \cdot 13$ & $649.89^{c}$ & 9.98 \\
\hline Malondialdehyde (nmol/ml) & $43.65^{\mathrm{a}}$ & 7.77 & $32 \cdot 80^{\mathrm{a}, \mathrm{b}}$ & 8.88 & $28 \cdot 17^{\mathrm{b}}$ & 1.02 & $24 \cdot 10^{\mathrm{b}}$ & 3.02 \\
\hline Lysozyme (U/ml) & $1.85^{\mathrm{b}}$ & 0.81 & $3 \cdot 21^{a, b}$ & 0.19 & $3.74^{\mathrm{a}}$ & 1.40 & $3.85^{\mathrm{a}}$ & 0.75 \\
\hline
\end{tabular}

a,b,c Mean values with unlike superscript letters were significantly different $(P<0.05)$. 
(A)
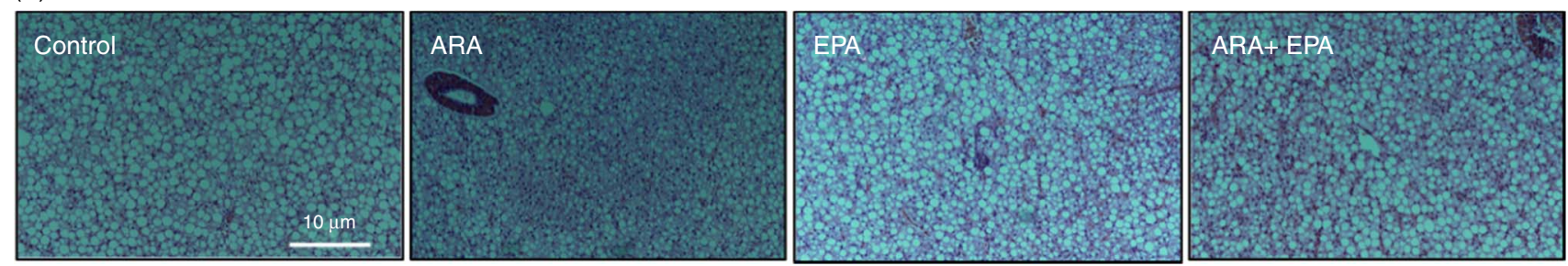

(B)
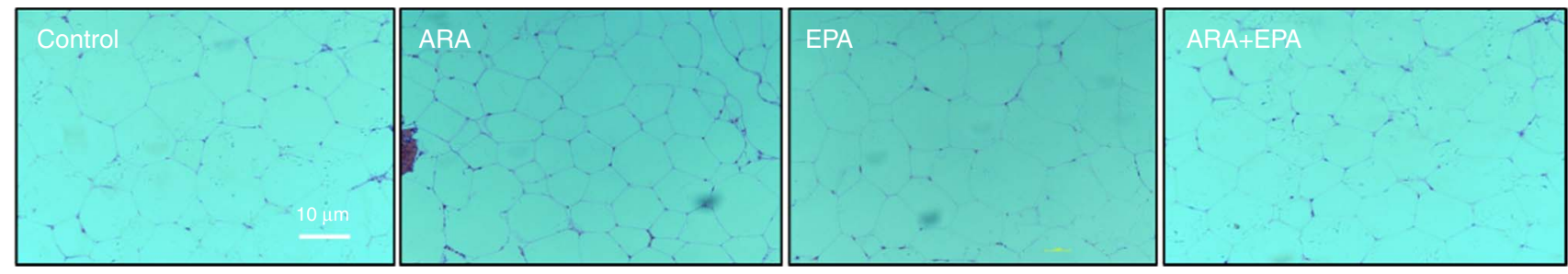

(C)
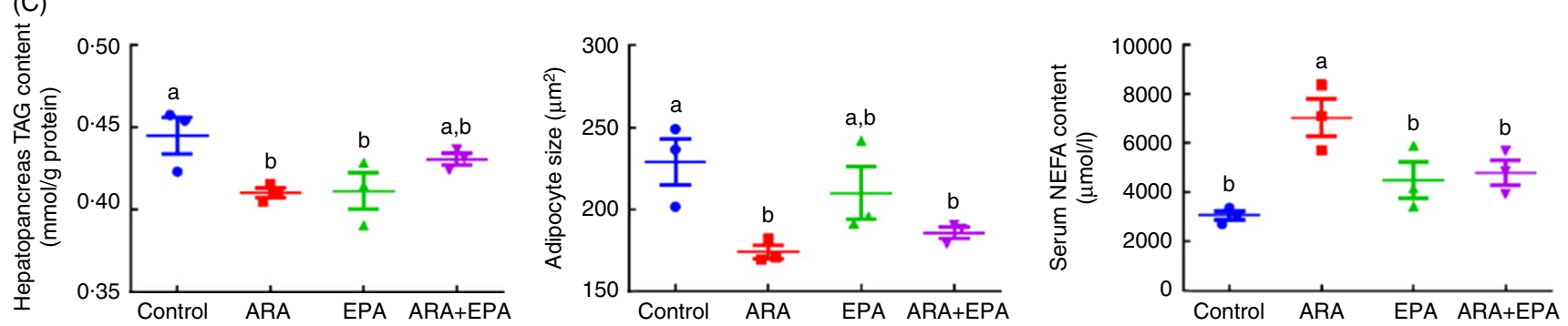

Fig. 2. Effects of dietary arachidonic acid (ARA) and EPA on the lipid accumulation in juvenile grass carp. (A) Histological morphology of the hepatopancreas; (B) histological morphology of intraperitoneal fat; (C) TAG content of hepatopancreas, adipocyte size, serum NEFA levels. Values are means ( $n$ 3), and standard deviations represented by vertical bars. ${ }^{a, b}$ Mean values with unlike letters were significantly different $(P<0.05)$.
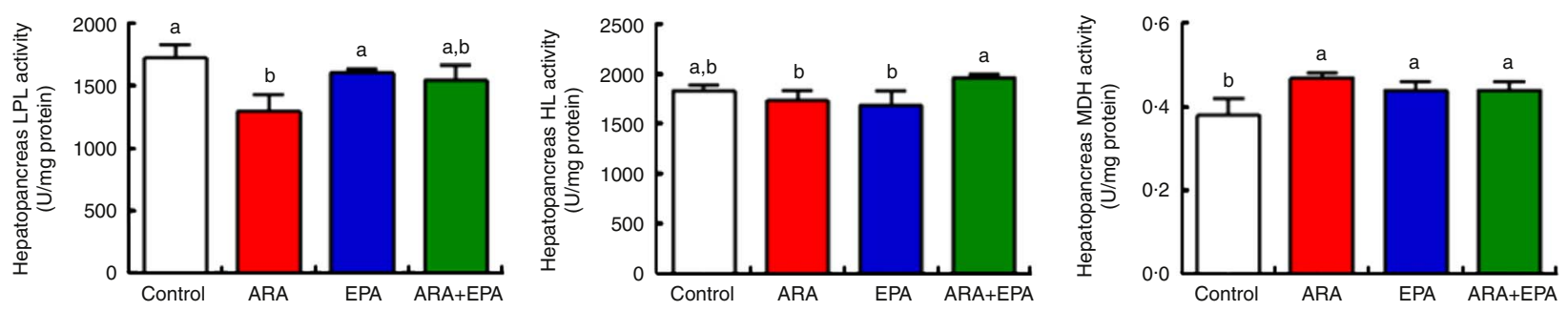

Fig. 3. Effects of dietary arachidonic acid (ARA) and EPA on the activities of lipid-metabolism-related enzymes in the hepatopancreas of juvenile grass carp. Values are means $(n 3)$, and standard deviations represented by vertical bars. LPL, lipoprotein lipase; HL, hepatic lipase; MDH, malate dehydrogenase. a,b Mean values with unlike letters were significantly different $(P<0.05)$.

$0 \cdot 3 \%$ EPA. The result that EPA promoted slightly higher growth than ARA has also been found in Atlantic salmon (Salmo salar) ${ }^{(30,31)}$. Actually, moderate dietary $n-3$ LC-PUFA (approximately $0.3 \%$ EPA and approximately $0.2 \%$ DHA) has been reported to increase growth in juvenile grass carp, suggesting that EPA concentration at $0 \cdot 3 \%$ or a high ratio of $n-3: n-6$ LC-PUFA might benefit the growth of juvenile grass carp ${ }^{(3)}$. The decreased $\mathrm{HI}$ in fish fed ARA, EPA or a combination of ARA and EPA was consistent with lipid accumulation in the hepatopancreas. These results highlighted the growth-promoting effects of dietary supplementation of EPA compared with ARA.

We also demonstrated different accumulation patterns of EPA and ARA in the hepatopancreas and IPF. As a prerequisite, given that the fatty acid compositions of hepatopancreas and IPF reflected those of the diets, dietary ARA and EPA were effectively incorporated into the fish lipid. In the hepatopancreas, ARA and EPA are more likely to retain in PL; in particular, ARA content was 7-fold higher in PL than that in NPL. A similar result was reported in the hepatopancreas, muscle and gill in gilthead bream (Sparusaurata L.) ${ }^{(14)}$. However, in IPF there was no apparent preference of these two fatty acids on NPL and PL, suggesting that adipose tissue might be an 'inert organ' that has lower ability for allotting ARA and EPA at a subcellular level. In the ARA + EPA group, EPA showed less levels than ARA did in tissues, although ARA and EPA were equally provided in the diet. This might be due to the conversion of EPA to DPA and 

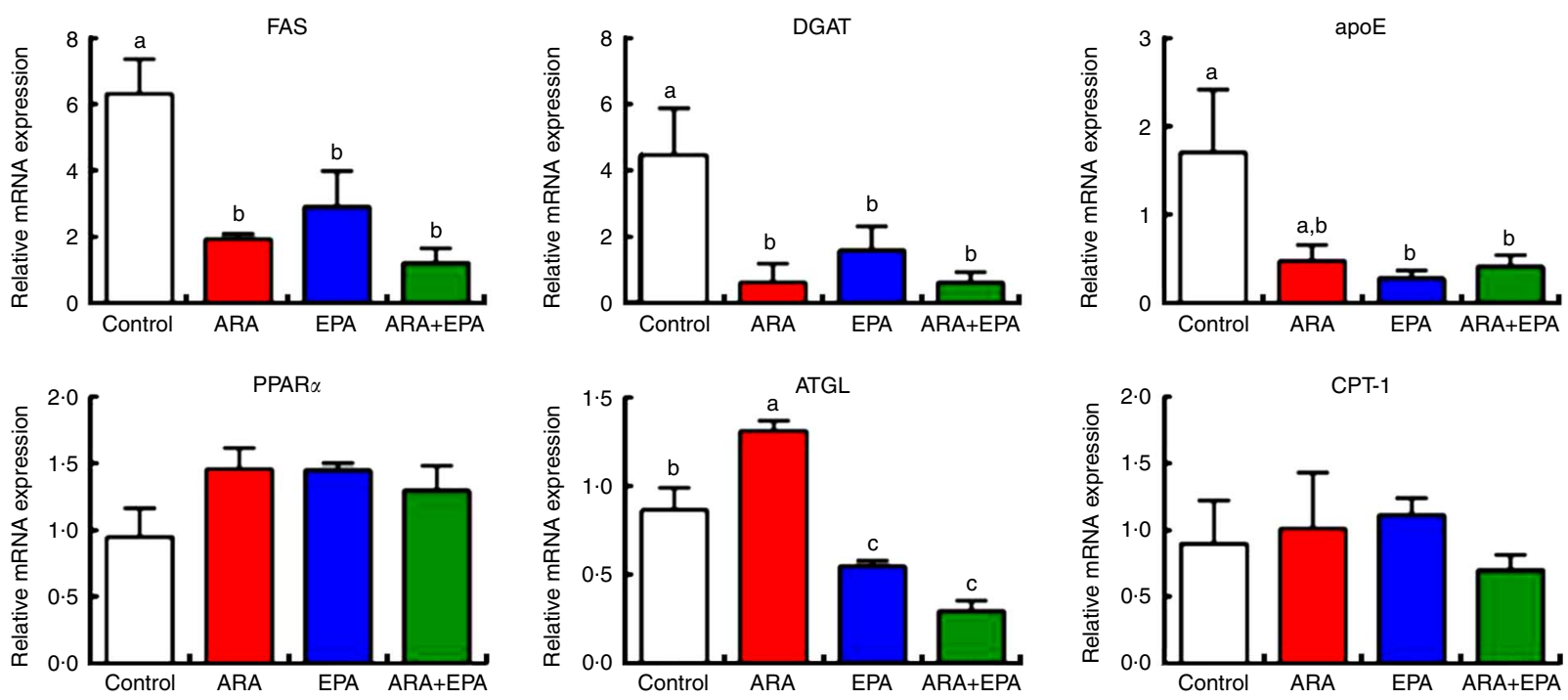

Fig. 4. Effects of dietary arachidonic acid (ARA) and EPA on the lipid-metabolism-related gene expression of the hepatopancreas in juvenile grass carp. Values are means $(n)$, and standard deviations represented by vertical bars. FAS, fatty acid synthase; DGAT, diacylglycerol O-acyltransferase; ATGL, adipose TAG lipase; CPT-1, carnitine palmitoyltransferase 1. ${ }^{\mathrm{a}, \mathrm{b}, \mathrm{c}}$ Mean values with unlike letters were significantly different $(P<0.05)$.
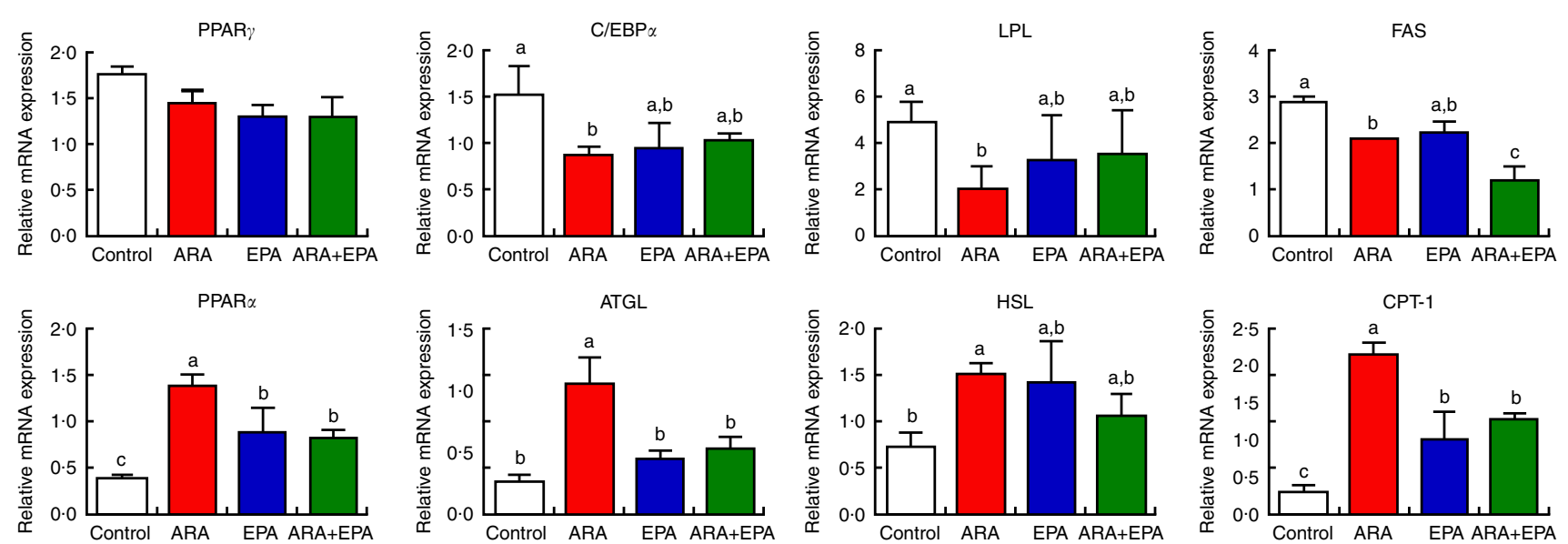

Fig. 5. Effects of dietary arachidonic acid (ARA) and EPA on the lipid-metabolism-related gene expression of intraperitoneal fat in juvenile grass carp. Values are means ( $n 3$ ), and standard deviations represented by vertical bars. C/EBPa, CCAAT enhancer-binding protein a; LPL, lipoprotein lipase; fatty acid synthase; ATGL, adipose TAG lipase; HSL, hormone-sensitive lipase; CPT-1, carnitine palmitoyltransferase 1 . $^{\text {a,b,c }}$ Mean values with unlike letters were significantly different $(P<0.05)$.

DHA, as suggested by the higher content of these two derivatives in fish fed EPA. There was a slightly inverse relationship between ARA and EPA content in hepatopancreas, but no such phenomenon was found in IPF. This was not consistent with early studies, which have observed an apparent decrease in EPA with increased dietary ARA levels in gilthead bream and Atlantic salmon $^{(14,48,49)}$, possibly because of the difference in fish species. It has been suggested that SFA and MUFA are preferentially located in the sn- 1 and sn- 3 position of the TAG and sn- 1 in the phosphoglycerides, whereas PUFA are preferentially located in the sn-2 position ${ }^{(11)}$. Roughly similarly, the fatty acid composition and PCA scores demonstrated that SFA and LC-PUFA are readily retained in PL, whereas MUFA is retained in NPL, suggesting that SFA have more affinity in PL and MUFA in NPL in grass carp. Besides, $\mathrm{C}_{18}$ PUFA presented higher content in IPF than in hepatopancreas. This might be because IPF is an 'entrepot' that accumulates dietary lipid for energy storage, whereas hepatopancreas is an important intermediary metabolism tissue that has higher capacity for fatty acid metabolism such as desaturation/ elongation and $\beta$-oxidation ${ }^{(39,50)}$

Because of the high unsaturation, ARA and EPA are easy to be attacked by free radicals, which cause lipid peroxidation and impair the functional integrity of cell membranes and enzyme activity ${ }^{(51,52)}$. MDA is the end product of lipid peroxidation and its content could reflect the degree of lipid peroxidation ${ }^{(53)}$. SOD is considered to play a pivotal antioxidant role and catalyses $\mathrm{O}_{2}^{-}$to $\mathrm{H}_{2} \mathrm{O}_{2}$, and $\mathrm{H}_{2} \mathrm{O}_{2}$ can be removed by the activities of CAT or GSH-Px ${ }^{(6)}$. It has been shown that moderate dietary ARA could increase the antioxidant response in fish such as Japanese seabass (Lateolabrax japonicus) ${ }^{(19)}$, Synechogobius hasta ${ }^{(54)}$ and Japanese eel (Anguilla japonica) ${ }^{(21)}$. Similar results have also been found in fish fed appropriate $n-3$ LC-PUFA $^{(35,55)}$. 
However, high levels of dietary ARA or $n$-3 LC-PUFA were reported to cause severe oxidative stress ${ }^{(3,19,54,56)}$. In the present study, we have shown that dietary $0.3 \%$ ARA and EPA decreased the serum MDA content, and increased the SOD and CAT activities, suggesting that these two fatty acids improved the antioxidant status at this concentration in diet of grass carp. Interestingly, GSH-Px activities were decreased in fish fed ARA and EPA, possibly because of an adaptive down-regulation of this enzyme resulting from lower free-radical levels. Our study also demonstrated that ARA and EPA increased the LZM activity, similar to the study in Japanese seabass ${ }^{(19)}$, Japanese eel ${ }^{(21)}$ and large yellow croaker (Larmichthyscrocea) ${ }^{(55)}$. Overall, these results demonstrate that ARA and EPA exhibited analogous function in regard to the antioxidant and antibacterial response.

In early studies, it was observed that dietary ARA decrease the lipid accumulation in grass carp ${ }^{(4,32,33)}$ as in other fish species $^{(14,19,54)}$. On the other side, considerable studies have shown that $n-3$ LC-PUFA, including EPA, have the capacity to decrease lipid content in fish, including grass carp ${ }^{(3,35,36,57)}$. In the present study, we have shown that $0.3 \%$ ARA and EPA decreased the TAG level in the hepatopancreas, whereas a combination of ARA and EPA had no such function, which might be due to the low concentration or the competition for binding enzymes. However, in regard to the dietary $n-6: n-3$ LC-PUFA ratios, high (ARA group, 8.2) or low (EPA group, $0 \cdot 10$ ) ratios presented a positive relationship with the inhibition of lipid accumulation compared with the moderate ratios (control group, 0.88; ARA + EPA group, 1.09), indicating that an imbalance of $n-6$ and $n-3$ LC-PUFA might result in the decrease of lipid retention in hepatopancreas, but this needs further confirmation. Interestingly, regarding the adipose tissue, although ARA blocked the development of adipocyte as indicated by the IPFI and adipocyte sizes, EPA had no such efficacy, suggesting that dietary EPA had weaker ability in repressing adipogenesis than ARA in this study. Taken together, these results demonstrated that dietary ARA and EPA played similar roles in repressing lipid accumulation in the hepatopancreas but only ARA acted as the inhibitor of adipose tissue development. Consistent with the possible inhibitory effects on adipogenesis, ARA but not EPA increased the serum NEFA level, possibly because of the up-regulation of the lipolytic genes in adipose tissue ${ }^{(58)}$

LPL is considered as a key enzyme in the lipid accumulation and metabolism of many tissues, which participates in the removal of lipoprotein TAG from the circulation (mainly chylomicron and very LDL) ${ }^{(59,60)}$. A decrease in LPL activity or gene expression with increasing dietary ARA has been suggested in S. hasta and grass carp ${ }^{(33,54)}$, which was also shown in this study. The low LPL activity was consistent with the low TAG level in hepatopancreas. EPA also inhibited this enzyme but to a lesser extent, and our early study has observed an increase in LPL activity in grass carp fed $n$ - 3 LC-PUFA. The effects of PUFA on LPL-mediated lipid metabolism might need further investigation $^{(3)}$. HL is primarily synthesised in liver and involved in chylomicron-remnant and HDL metabolism ${ }^{(61)}$. It should be noted that the ARA + EPA group presented significantly higher HL activity, which might be because of the moderate ratio of $n-6: n-3$ LC-PUFA and may possibly be the reason for the relatively higher lipid accumulation in this group. MDH plays a key part in the malate/aspartate shuttle across the mitochondrial membrane and in the tricarboxylic acid cycle within the mitochondrial matrix ${ }^{(62)}$. In our study, dietary ARA, EPA and a combination of ARA and EPA increased the hepatopancreatic $\mathrm{MDH}$ activities, indicating that these $\mathrm{C}_{20}$ LC-PUFA could increase the active status of tricarboxylic acid cycle.

In this study, both ARA and EPA decreased the de novo lipogenesis capacity in a similar manner as suggested by the down-regulation of FAS and DGAT mRNAs in hepatopancreas, which is consistent with the low TAG levels. Many studies have demonstrated that ARA and $n$-3 LC-PUFA could decrease the lipogenic gene expression in grass $\operatorname{carp}^{(4,33,35,36)}$. ApoE is mainly produced by the liver and combines with lipids in the body to form lipoproteins, acting as a key regulator of plasma lipid levels ${ }^{(63)}$. Interestingly, all dietary LC-PUFA treatments resulted in the decrease of the expression of $a p o E$ in this study, suggesting a lower capacity of packaging lipoproteins in these groups. However, these gene expressions seem to have no relevance to the ratio of $n-3: n-6$ LC-PUFA, as proved in the ARA + EPA group. A clear difference between ARA and EPA was found in lipid catabolism, which is also an important factor influencing lipid accumulation. Only dietary ARA increased the expression of $A T G L$ that catalyses the first step for the sequential hydrolysis of $\mathrm{TAG}^{(64)}$. There is one previous study showing similar results ${ }^{(33)}$. Considering that the hepatopancreatic TAG contents were similar in the ARA and EPA groups, lipid anabolism would play the primary role in controlling TAG level in hepatopancreas. Alternatively, compared with EPA, dietary ARA may decrease lipid incorporation and enhance mobilisation in the hepatopancreas (as suggested by LPL activity and $A T G L$ mRNA levels), resulting in the apparently similar TAG levels.

ARA decreased the expression of the adipogenic marker genes $C / E B P \alpha, L P L$ and $F A S$, and increased lipid catabolic mRNA levels of PPAR $\alpha, A T G L, H S L$ and $C P T-1$ in IPF. The EPA group showed less effects on these genes, although it has been suggested that EPA also could trigger the inhibition of adipogenesis and promotion of lipolysis ${ }^{(34,35,57)}$. The increased serum NEFA levels in fish fed ARA but not EPA also demonstrated the higher lipolysis-enhancing effect of ARA compared with EPA. In the present study, EPA level in the IPF was less than that in ARA, partly because of the conversion to DPA or DHA. The low EPA concentration might account for its low influence on lipid metabolism genes. In another study, we observed that EPA content at $0.6 \%$ could effectively inhibit lipogenic and increase lipolytic genes (unpublished results). Moreover, it has been suggested that the metabolites of ARA from the COX pathway might be responsible for the improvement of lipid catabolic capacity in grass carp ${ }^{(32,33)}$. The EPA-derived COX eicosanoids are less active possibly because of the lower level of substrate $^{(6,11)}$. It is difficult in in vivo studies to exactly match intracellular levels of the target fatty acids. Detailed mechanisms underlying the difference between ARA and EPA will be addressed in further in vitro studies using model systems.

In conclusion, the present result showed that diet supplemented with EPA was beneficial on the growth performance, whereas ARA exhibited more potent ability in controlling lipid metabolism in IPF. These fatty acids showed similar effects on 
lipid metabolism in the hepatopancreas. ARA and EPA are more likely to be esterified in PL in the hepatopancreas than in IPF, and presented similar effects on the antioxidant capacity.

\section{Acknowledgements}

The authors thank Ankang Fisheries Experimental and Demonstration Station of the Northwest Agriculture and Forestry University for the experimental fish.

This study was supported by the National Natural Science Foundation of China (grant nos 31372538, K3050215105) and the earmarked fund for China Agriculture Research System (grant no. CARS-46-16). The funders had no role in the design, analysis or writing of the article.

J.-j. T., H. J. and J. X. conceived and designed the experiments. J.-j. T. and C.-X. L. performed the experiments and contributed to the analysis of data. J.-j. T. and G. K. co-wrote the manuscript. C.-X. L., H. J., J.-s. Z., H.b. Y., Y. L. and E.-m. Y. contributed to the revision.

The authors declare that there are no conflicts of interest.

\section{References}

1. Higgs D \& Dong F (2000) Lipids and fatty acids. In Encyclopedia of Aquaculture, pp. 476-496 [RR Stickney, editor]. New York: John Wiley and Sons.

2. Kiron V, Thawonsuwan J, Panigrahi A, et al. (2011) Antioxidant and immune defences of rainbow trout (Oncorbynchus mykiss) offered plant oils differing in fatty acid profiles from early stages. Aquacult Nutr 17, 130-140.

3. Ji H, Li J \& Liu P (2011) Regulation of growth performance and lipid metabolism by dietary $n-3$ highly unsaturated fatty acids in juvenile grass carp, Ctenopharyngodon idellus. Comp Biochem Physiol B Biochem Mol Biol 159, 49-56.

4. Tian J, Ji H, Oku H, et al. (2014) Effects of dietary arachidonic acid (ARA) on lipid metabolism and health status of juvenile grass carp, Ctenopharyngodon idellus. Aquaculture 430 , $57-65$.

5. Bell JG \& Sargent JR (2003) Arachidonic acid in aquaculture feeds: current status and future opportunities. Aquaculture 218, 491-499.

6. Tocher DR (2003) Metabolism and functions of lipids and fatty acids in teleost fish. Rev Fish Sci 11, 107-184.

7. Yin H, Zhou Y, Zhu M, et al. (2013) Role of mitochondria in programmed cell death mediated by arachidonic acid-derived eicosanoids. Mitochondrion 13, 209-224.

8. Funk CD (2001) Prostaglandins and leukotrienes: advances in eicosanoid biology. Science 294, 1871-1875.

9. Wang D \& DuBois RN (2010) Eicosanoids and cancer. Nat Rev Cancer 10, 181-193.

10. Hamre K, Moren M, Solbakken J, et al. (2005) The impact of nutrition on metamorphosis in Atlantic halibut (Hippoglossus hippoglossus L.). Aquaculture 250, 555-565.

11. Halver JE \& Hardy RW (2002) Fish Nutrition, 3rd ed. San Diego, CA: Academic Press.

12. Moreno JJ (2009) Differential effects of arachidonic and eicosapentaenoic acid-derived eicosanoids on polymorphonuclear transmigration across endothelial cell cultures. J Pharmacol Exp Ther 331, 1111-1117.

13. Rodríguez-Lagunas MJ, Ferrer R \& Moreno JJ (2013) Effect of eicosapentaenoic acid-derived prostaglandin $\mathrm{E}_{3}$ on intestinal epithelial barrier function. Prostaglandins Leukot Essent Fatty Acids 88, 339-345.

14. Fountoulaki E, Alexis M, Nengas I, et al. (2003) Effects of dietary arachidonic acid (20:4n-6), on growth, body composition, and tissue fatty acid profile of gilthead bream fingerlings (Sparus aurata L.). Aquaculture 225, 309-323.

15. Atalah E, Hernández-Cruz CM, Ganuza E, et al. (2011) Importance of dietary arachidonic acid for the growth, survival and stress resistance of larval European sea bass (Dicentrarchus labrax) fed high dietary docosahexaenoic and eicosapentaenoic acids. Aquacult Res 42, 1261-1268.

16. Carrier JK III, Watanabe WO, Harel M, et al. (2011) Effects of dietary arachidonic acid on larval performance, fatty acid profiles, stress resistance, and expression of $\mathrm{Na}^{+} / \mathrm{K}^{+}$ATPase mRNA in black sea bass Centropristis striata. Aquaculture 319, 111-121.

17. Rezek TC, Watanabe WO, Harel M, et al. (2010) Effects of dietary docosahexaenoic acid $(22: 6 n-3)$ and arachidonic acid $(20: 4 n-6)$ on the growth, survival, stress resistance and fatty acid composition in black sea bass Centropristis striata (Linnaeus 1758) larvae. Aquacult Res 41, 1302-1314.

18. Montero D, Terova G, Rimoldi S, et al. (2015) Modulation of the expression of components of the stress response by dietary arachidonic acid in European sea bass (Dicentrarchus labrax) larvae. Lipids 50, 1029-1041.

19. Xu H, Ai Q, Mai K, et al. (2010) Effects of dietary arachidonic acid on growth performance, survival, immune response and tissue fatty acid composition of juvenile Japanese seabass, Lateolabrax japonicus. Aquaculture 307, 75-82.

20. Furne M, Holen E, Araujo P, et al. (2013) Cytokine gene expression and prostaglandin production in head kidney leukocytes isolated from Atlantic cod (Gadus morbua) added different levels of arachidonic acid and eicosapentaenoic acid. Fish Shellfish Immunol 34, 770-777.

21. Shahkar E, Yun H, Lee S, et al. (2016) Evaluation of the optimum dietary arachidonic acid level and its essentiality based on growth and non-specific immune responses in Japanese eel, Anguilla japonica. Aquaculture 452, 209-216.

22. Norambuena F, Estévez A, Mañanós E, et al. (2013) Effects of graded levels of arachidonic acid on the reproductive physiology of Senegalese sole (Solea senegalensis): fatty acid composition, prostaglandins and steroid levels in the blood of broodstock bred in captivity. Gen Comp Endocrinol 191, 92-101.

23. Norambuena F, Morais S, Estévez A, et al. (2013) Dietary modulation of arachidonic acid metabolism in senegalese sole (Solea Senegalensis) broodstock reared in captivity. Aquaculture 372, 80-88.

24. Xu H, Cao L, Zhang Y, et al. (2017) Dietary arachidonic acid differentially regulates the gonadal steroidogenesis in the marine teleost, tongue sole (Cynoglossus semilaevis), depending on fish gender and maturation stage. Aquaculture 468, 378-385.

25. Villalta M, Estévez A \& Bransden MP (2005) Arachidonic acid enriched live prey induces albinism in Senegal sole (Solea senegalensis) larvae. Aquaculture 245, 193-209.

26. Boglino A, Wishkerman A, Darias MJ, et al. (2014) Senegalese sole (Solea senegalensis) metamorphic larvae are more sensitive to pseudo-albinism induced by high dietary arachidonic acid levels than post-metamorphic larvae. Aquaculture $\mathbf{4 3 3}$, 276-287.

27. Wishkerman A, Boglino A, Darias M J, et al. (2016) Image analysis-based classification of pigmentation patterns in fish: a case study of pseudo-albinism in Senegalese sole. Aquaculture 464, 303-308.

28. Lie KK, Kvalheim K, Rasinger JD, et al. (2016) Vitamin A and arachidonic acid altered the skeletal mineralization in Atlantic 
cod (Gadus morbua) larvae without any interactions on the transcriptional level. Comp Biochem Physiol A Mol Integr Physiol 191, 80-88.

29. Boglino A, Darias MJ, Estévez A, et al. (2012) The effect of dietary arachidonic acid during the Artemia feeding period on larval growth and skeletogenesis in Senegalese sole, Solea senegalensis. J Appl Ichthyol 28, 411-418.

30. Norambuena F, Morais S, Emery JA, et al. (2015) Arachidonic acid and eicosapentaenoic acid metabolism in juvenile Atlantic salmon as affected by water temperature. PLOS ONE 10, e0143622.

31. Norambuena F, Rombenso A \& Turchini GM (2016) Towards the optimization of performance of Atlantic salmon reared at different water temperatures via the manipulation of dietary ARA/EPA ratio. Aquaculture 450, 48-57.

32. Tian JJ, Lei CX, Ji H, et al. (2016) Dietary arachidonic acid has a time-dependent differential impact on adipogenesis modulated via COX and LOX pathways in Grass carp Ctenopharyngodon idellus. Lipids 51, 1325-1338.

33. Tian JJ, Lei CX, Ji H, et al. (2017) Role of cyclooxygenasemediated metabolites in lipid metabolism and expression of some immune-related genes in juvenile grass carp (Ctenopharyngodon idellus) fed arachidonic acid. Fish Physiol Biochem 43, 703-717.

34. Liu P, Li C, Huang J, et al. (2014) Regulation of adipocytes lipolysis by $n$ - 3 HUFA in grass carp (Ctenopharyngodon idellus) in vitro and in vivo. Fish Physiol Biochem 40, 1447-1460.

35. Li C, Liu P, Ji H, et al. (2015) Dietary $n$-3 highly unsaturated fatty acids affect the biological and serum biochemical parameters, tissue fatty acid profile, antioxidation status and expression of lipid-metabolism-related genes in grass carp, Ctenopharyngodon idellus. Aquacult Nutr 21, 373-383.

36. Tian JJ, Lu RH, Ji H, et al. (2015) Comparative analysis of the hepatopancreas transcriptome of grass carp (Ctenopharyngodon idellus) fed with lard oil and fish oil diets. Gene $\mathbf{5 6 5}$, 192-200.

37. Glencross BD (2009) Exploring the nutritional demand for essential fatty acids by aquaculture species. Rev Aquacult 1, 71-124.

38. Tocher DR (2010) Fatty acid requirements in ontogeny of marine and freshwater fish. Aquacult Res 41, 717-732.

39. Tian JJ, Lei CX \& Ji H (2016) Influence of dietary linoleic acid (18:2n-6) and $\alpha$-linolenic acid $(18: 3 n-3)$ ratio on fatty acid composition of different tissues in freshwater fish Songpu mirror carp, Cyprinus carpio. Aquacult Res 47, 3811-3825.

40. Lavell R (1989) Nutrition and Feeding of Fish. New York: Auburn University.

41. Association of Official Analytical Chemists (1995) Official Methods of Analysis of Official Analytical Chemists International, 16th ed. Arlington, VA: Association of Official Analytical.

42. Folch J, Lees M \& Sloane Stanley G (1957) A simple method for the isolation and purification of total lipides from animal tissues. J Biol Chem 226, 497-509.

43. Juaneda P \& Rocquelin G (1985) Rapid and convenient separation of phospholipids and non phosphorus lipids from rat heart using silica cartridges. Lipids 20, 40-41.

44. Liu P, Ji H, Li C, et al. (2015) Ontogenetic development of adipose tissue in grass carp (Ctenopharyngodon idellus). Fish Phys Biochem 41, 867-878.

45. Osman OS, Selway JL, Kępczyńska MA, et al. (2013) A novel automated image analysis method for accurate adipocyte quantification. Adipocyte 2, 160-164.
46. Livak KJ \& Schmittgen TD (2001) Analysis of relative gene expression data using real-time quantitative PCR and the $2^{-\Delta \Delta C T}$ method. Methods 25, 402-408.

47. Pfaffl MW (2001) A new mathematical model for relative quantification in real-time RT-PCR. Nucleic Acids Res 29, e45-e45.

48. Bessonart M, Izquierdo MS, Salhi M, et al. (1999) Effect of dietary arachidonic acid levels on growth and survival of gilthead sea bream (Sparus aurata L.) larvae. Aquaculture 179, 265-275.

49. Bell JG, McVicar AH, Park MT, et al. (1991) High dietary linoleic acid affects the fatty acid compositions of individual phospholipids from tissues of Atlantic salmon (Salmo salar): association with stress susceptibility and cardiac lesion. J Nutr 121, 1163-1172.

50. Turchini GM, Torstensen BE \& Ng WK (2009) Fish oil replacement in finfish nutrition. Rev Aquacult 1, 10-57.

51. Gray JI (1978) Measurement of lipid oxidation: a review. J Am Oil Chem Soc 55, 539-546.

52. Halliwell B \& Gutteridge JM (2015) Free Radicals in Biology and Medicine. New York: Oxford University Press.

53. Surapaneni K \& Venkataramana G (2007) Status of lipid peroxidation, glutathione, ascorbic acid, vitamin $\mathrm{E}$ and antioxidant enzymes in patients with osteoarthritis. Indian J Med Sci $\mathbf{6 1}$, 9-14.

54. Luo Z, Tan XY, Li XD, et al. (2012) Effect of dietary arachidonic acid levels on growth performance, hepatic fatty acid profile, intermediary metabolism and antioxidant responses for juvenile Synechogobius hasta. Aquacult Nutr 18, 340-348.

55. Zuo R, Ai Q, Mai K, et al. (2012) Effects of dietary $n$-3 highly unsaturated fatty acids on growth, nonspecific immunity, expression of some immune related genes and disease resistance of large yellow croaker (Larmichthys crocea) following natural infestation of parasites (Cryptocaryon irritans). Fish Shellfish Immunol 32, 249-258.

56. Todorčević M, Kjær MA, Djaković N, et al. (2009) n-3 HUFAs affect fat deposition, susceptibility to oxidative stress, and apoptosis in Atlantic salmon visceral adipose tissue. Comp Biochem Physiol B Biochem Mol Biol 152, 135-143.

57. Todorčević M \& Hodson L (2015) The effect of marine derived $n-3$ fatty acids on adipose tissue metabolism and function. J Clin Med 5, 3.

58. Frühbeck G, Méndez-Giménez L, Fernández-Formoso JA, et al. (2014) Regulation of adipocyte lipolysis. Nutr Res Rev 27, 63-93.

59. Eckel RH (1989) Lipoprotein lipase. New Engl J Med 320, 1060-1068.

60. Nilsson-Ehle P, Garfinkel AS \& Schotz MC (1980) Lipolytic enzymes and plasma lipoprotein metabolism. Annu Rev Biochem 49, 667-693.

61. Connelly PW (1999) The role of hepatic lipase in lipoprotein metabolism. Clin Chim Acta 286, 243-255.

62. Minarik P, Tomaskova N, Kollarova M, et al. (2002) Malate dehydrogenases-structure and function. Gen Physiol Biophys 21, 257-266

63. Phillips MC (2014) Apolipoprotein E isoforms and lipoprotein metabolism. IUBMB Life 66, 616-623.

64. Sun J, Ji H, Li XX, et al. (2016) Lipolytic enzymes involving lipolysis in teleost: synteny, structure, tissue distribution, and expression in grass carp (Ctenopharyngodon idella). Comp Biochem Physiol B Biochem Mol Biol 198, 110-118. 